\title{
Um modelo de evolução de espécies com extinções em massa
}

\author{
Fábio Sternieri Marques
}

\author{
DISSERTAÇÃO APRESENTADA \\ $\mathrm{AO}$ \\ Instituto DE MATEMÁticA E EstatísticA \\ DA \\ UNIVERSIDADE DE SÃO PAUlO \\ PARA \\ OBTENÇÃO DO TÍTULO \\ DE \\ Mestre EM CiÊnCIAS
}

Programa: Estatística

Orientador: Prof. Dr. Luiz Renato Gonçalves Fontes

Durante o desenvolvimento deste trabalho o autor recebeu auxílio financeiro do CNPq 


\section{Um modelo de evolução de espécies com extinções em massa}

Esta versão da dissertação contém as correções e alterações sugeridas pela Comissão Julgadora durante a defesa da versão original do trabalho, realizada em 23/04/2018. Uma cópia da versão original está disponível no

Instituto de Matemática e Estatística da Universidade de São Paulo.

Comissão Julgadora:

- Prof. Dr. Luiz Renato Gonçalves Fontes (orientador) - IME/USP

- Prof a Dr a . Carolina Bueno Grejo - Pós-Doc ICMC/USP

- Prof. Dr. Élcio Lebenstayn - UNICAMP 


\section{Resumo}

Marques, F. S. Um modelo de evolução de espécies com extinções em massa. 2018. $31 \mathrm{f}$. Dissertação (Mestrado) - Instituto de Matemática e Estatística, Universidade de São Paulo, São Paulo, 2018.

Apresentamos um modelo estocástico para evolução de espécies utilizando processos de Poisson. Eventos de surgimento de novas espécies e eventos de extinção são dados por dois processos de Poisson independentes. A cada evento de surgimento, uma nova espécie é adicionada ao sistema e uma aptidão aleatória é associada a ela. À cada evento de extinção, é associado um limiar também aleatório e todas as espécies com aptidão inferior ao limiar são retiradas do sistema. Apresentamos critérios necessários e suficientes para recorrência/transitoriedade da configuração vazia. Mostramos a existência da distribuição limite e apresentamos critérios necessários e suficientes para um número in/finito de espécies em tal distribuição.

Palavras-chave: Processos de Poisson, Recordes, Evolução. 


\section{Abstract}

Marques, F. S. A species evolution model with mass extinction. 2018. 31 f. Dissertação (Mestrado) - Instituto de Matemática e Estatística, Universidade de São Paulo, São Paulo, 2018.

We present a stochastic model for species evolution based on Poisson Processes. Birth of new species and extinction events are given by two independent Poisson processes. At each birth, a new species enters the system and is given a random fitness mark. To each extinction event is associated a random threshold mark and all species with fitness lower than the threshold are removed from the system. We present necessary and suficient criteria for the recurrence/transience of the empty configuration. We show the existence of the limit distribution and present necessary and suficient criteria for an in/finite number os species in such distribution.

Keywords: Poisson Processes, Records, Evolution. 


\section{Sumário}

1 Introdução $\quad \mathbf{1}$

1.1 Apresentação do modelo . . . . . . . . . . . . . . . . . . . . . . . 1

1.2 Relação com o modelo GMS . . . . . . . . . . . . . . . . . . . . . . . . . . . . . . . . . . . . . . . . .

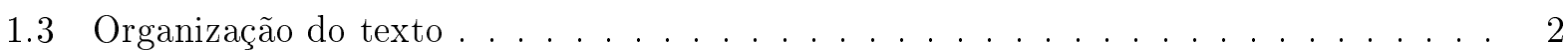

2 Modelo e Resultados $\quad 3$

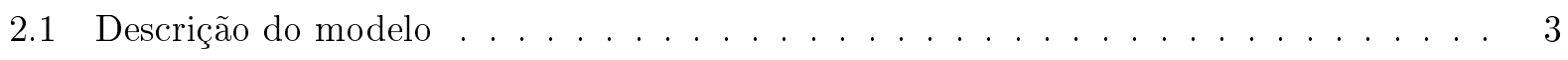

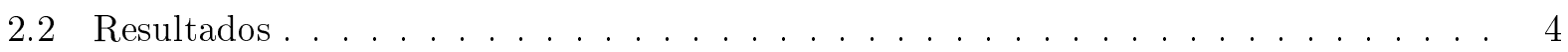

$\begin{array}{lll}3 & \text { Recorrência e Transitoriedade } & 7\end{array}$

3.1 Escada de aptidões recordes . . . . . . . . . . . . . . . . 7

3.2 Extinções acima da escada via funcional de Laplace . . . . . . . . . . . . . . . . . . 8

3.3 Finitude das extinções . . . . . . . . . . . . . . . . . . . . . . . . 10

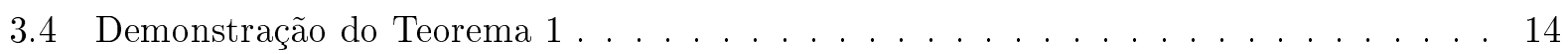

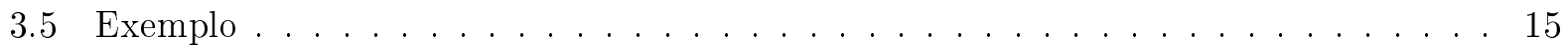

4 Distribuição Limite $\quad 17$

4.1 Escada de limiares recordes . . . . . . . . . . . . . . . . . . 17

4.2 Demonstração do Teorema $2 \ldots \ldots \ldots \ldots \ldots$

4.3 O número de espécies presentes na distribuição limite . . . . . . . . . . . . . . . . . . . . . . . . . . . . . . . . . .

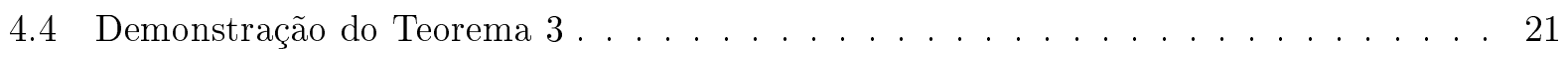

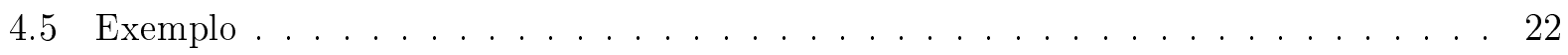

A Complementos $\quad 25$

A.1 Recordes como processo de Poisson . . . . . . . . . . . . . . . . 25

A.2 Mais alguns resultados utilizados . . . . . . . . . . . . 27

$\begin{array}{ll}\text { Referências Bibliográficas } & 31\end{array}$ 


\section{Capítulo 1}

\section{Introdução}

\subsection{Apresentação do modelo}

Nesta dissertação, apresentamos um modelo estocástico para evolução de espécies. Cada espécie é identificada por uma aptidão, que representa sua capacidade de sobreviver a eventos de extinção. Novas espécies surgem em instantes aleatórios, dados por um processo de Poisson. A cada evento de surgimento, uma única espécie é adicionada ao sistema e sua aptidão é selecionada aleatoriamente de uma distribuição $F_{\star}$, independente do instante de surgimento. Vamos assumir que tal distribuição é absolutamente contínua, o que garante aptidões distintas para cada espécie.

Eventos de extinção ocorrem em instantes dados por um segundo processo de Poisson, independente do primeiro. A cada evento de extinção, um limiar é selecionado aleatoriamente de uma distribuição $F_{\dagger}$, independente do instante em que ocorre. A cada evento de extinção, todas as espécies com aptidão inferior ao limiar são removidas do sistema. Um único evento de extinção poderia remover todas as espécies de uma única vez ou apenas as mais vulneráveis, dependendo do valor do limiar e da configuração de aptidões no instante do evento.

Vamos analisar duas questões. A primeira é a recorrência ou transitoriedade da configuração vazia. Apresentamos critérios necessários e suficientes, expressos em termos de processo de Poisson de recordes. Utilizamos técnicas como marcações e funcional de Laplace. A segunda questão é a existência de distribuição limite e o número de espécies presentes nesta distribuição. Para mostrar a convergência em distribuição, vamos analisar o processo no sentido inverso do tempo. Apresentamos critérios necessários e suficientes para a in/finitude do número de espécies na distribuição limite, utilizando as mesmas técnicas da primeira questão. Algumas das técnicas utilizadas podem ser encontradas no apêndice.

\subsection{Relação com o modelo GMS}

O modelo apresentado nesta dissertação é uma variante do modelo GMS, introduzido em Guiol et al. (2013). No modelo GMS, novas espécies são adicionadas ao sistema em instantes dados por um processo de Poisson. A cada evento de surgimento, uma aptidão é selecionada aleatoriamente de uma distribuição absolutamente contínua, independentemente do instante de surgimento. Assim, os surgimentos ocorrem da mesma forma nos dois modelos. Por outro lado, os eventos de extinção são um pouco diferentes. No modelo GMS, os eventos de extinção também ocorrem em instantes dados por um segundo processo de Poisson independente do primeiro, mas uma única espécie é removida do sistema a cada um destes eventos: aquela com a menor aptidão.

No modelo GMS, existe um valor crítico que divide as aptidões em duas fases. Esta aptidão crítica depende das taxas dos processos de Poisson e também da distribuição utilizada para selecionar as aptidões de novas espécies. As espécies presentes na fase subcrítica, aquelas com aptidão inferior ao valor crítico, são removidas do sistema em algum instante aleatório quase certamente finito. Já as espécies na fase supercrítica, aquelas com aptidões superiores ao valor crítico, têm probabilidade positiva de permanecerem no sistema indefinidamente. Estes resultados podem ser encontrados em 
Guiol et al. (2013). Os autores calculam a distribuição do tempo de sobrevivência de cada espécie e mostam que o número de espécies na fase subcrítica é um processo de nascimento e morte recorrente nulo. No modelo apresentado nesta dissertação, as hipóteses sobre as funções de distribuição garantem que cada espécie seja removida do sistema em tempo quase certamente finito.

A existência da distribuição limite para um caso particular do modelo GMS, com distribuição uniforme, foi analisada em Pinheiro (2015). O mesmo caso aparece em Formentin e Swart (2016) para um modelo de respostas de mensagens de email com prioridades. Neste caso, a distribuição limite tem infinitas espécies na fase subcrítica, mas com um único ponto de acumulação: a aptidão crítica. Outra questão abordada em Pinheiro (2015) é o surgimento de espécies eternas. O autor mostra que o tempo de surgimento da primeira espécie eterna na fase supercrítica é quase certamente finito. No modelo apresentado nesta dissertação, não existem espécies eternas, como já comentado acima.

\subsection{Organização do texto}

No Capítulo 2, apresentamos uma descrição do modelo e enunciamos os principais resultados. No Capítulo 3, analisamos a questão de recorrência/transitoriedade. No Capítulo 4, mostramos a existência da distribuição limite e analisamos o número de espécies presentes em tal distribuição. Incluímos também um apêndice com alguns resultados utilizados no texto, como marcações e funcional de Laplace. 


\section{Capítulo 2}

\section{Modelo e Resultados}

\subsection{Descrição do modelo}

Nesta seção, apresentamos uma descrição do modelo. Sejam $\Pi_{\star}$ e $\Pi_{\dagger}$ processos de Poisson em $\mathbb{R}$ homogêneos com taxas $\lambda_{\star}$ e $\lambda_{\dagger}$ respectivamente, independentes entre si e definidos no mesmo espaço de probabilidades $(\Omega, \mathcal{F}, \mathbb{P})$. Denotamos por $T_{i} \in \Pi_{\star}$ com $i \in \mathbb{Z}^{*}$ os instantes de surgimentos de novas espécies e por $S_{j} \in \Pi_{\dagger}$ com $j \in \mathbb{Z}^{*}$ os instantes dos eventos de extinção, em ordem crescente:

$$
\begin{aligned}
& \ldots<T_{-2}<T_{-1}<0<T_{1}<T_{2}<\ldots \\
& \ldots<S_{-2}<S_{-1}<0<S_{1}<S_{2}<\ldots
\end{aligned}
$$

Sejam $X$ e $Y$ variáveis aleatórias absolutamente contínuas com funções de distribuição $F_{\star}$ e $F_{\dagger}$, satisfazendo as seguintes condições: $F(x)=0$ para $x \leq 0$ (V.A. positiva), $F(x)<1$ para cada $x \in \mathbb{R}$ (V.A. ilimitada superiormente), $F$ é estritamente crescente e admite inversa. A cada instante $T_{i} \in \Pi_{\star}$ associamos uma aptidão $X_{i}$ usando a distribuição $F_{\star}$. A cada instante $S_{j} \in \Pi_{\dagger}$ associamos um limiar $Y_{j}$ usando a distribuição $F_{\dagger}$. Denotamos a cauda das distribuições por $\bar{F}(x)=1-F(x)$.

A seguir, vamos definir o processo $\eta_{t}=\left\{\eta_{t}(s), s \geq t\right\}$, que pode ser descrito em palavras da seguinte forma: no instante inicial $t \in \mathbb{R}$, o processo tem configuração inicial $\eta_{t}(t)=A$, onde $A$ é um subconjunto localmente finito de $\mathbb{R}^{+}$; no instante $s \in(t,+\infty)$, o processo tem configuração $\eta_{t}(s)$, um subconjunto localmente finito de $\mathbb{R}^{+}$, formada por todas as espécies que tenham surgido no intervalo $(t, s]$, ou pertencentes à configuração inicial, que tenham sobrevivido a cada extinção subsequente; ou seja, aquelas cuja aptidão seja superior ao limiar de extinção recorde associado à época de surgimento daquela espécie. As épocas são subintervalos de $(t, s]$ delimitados pelos instantes de ocorrência dos limiares recordes. Para as espécies da configuração inicial $A$, vale o limiar recorde da época mais antiga. Veja Figura 2.1.

Para cada $s \in \mathbb{R}$, defina os índices recordes $J_{k}(s)$ e valores recordes $Y_{J_{k}}(s)$ por:

$$
\begin{aligned}
J_{1}(s) & \doteq \max \left\{j: S_{j} \leq s\right\} \\
J_{k+1}(s) & \doteq \max \left\{j<J_{k}(s): Y_{j}>Y_{J_{k}}(s)\right\} \quad \text { para cada } \quad k \geq 1
\end{aligned}
$$

Vamos definir o processo $\eta_{t}=\left\{\eta_{t}(s), s \geq t\right\}$ para cada $t \in \mathbb{R}$. Dado um subconjunto localmente finito $A \subset \mathbb{R}^{+}$, defina a configuração inicial do processo por $\eta_{t}(t) \doteq A$. Para $s>t$, defina $\eta_{t}(s)$ por:

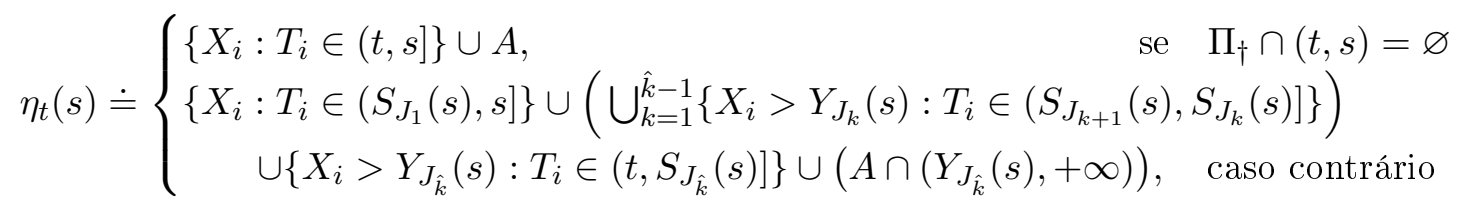

onde $\hat{k} \doteq \max \left\{k: S_{J_{k}}(s)>t\right\}$ e $\bigcup_{k=1}^{\hat{k}-1}\left\{X_{i}>Y_{J_{k}}(s): T_{i} \in\left(S_{J_{k+1}}(s), S_{J_{k}}(s)\right]\right\}=\varnothing$ se $\hat{k}=1$.

Em palavras, $\hat{k}$ é o número de extinções recordes no intervalo $(t, s)$ e também, o índice da extinção com o maior limiar no mesmo intervalo. 


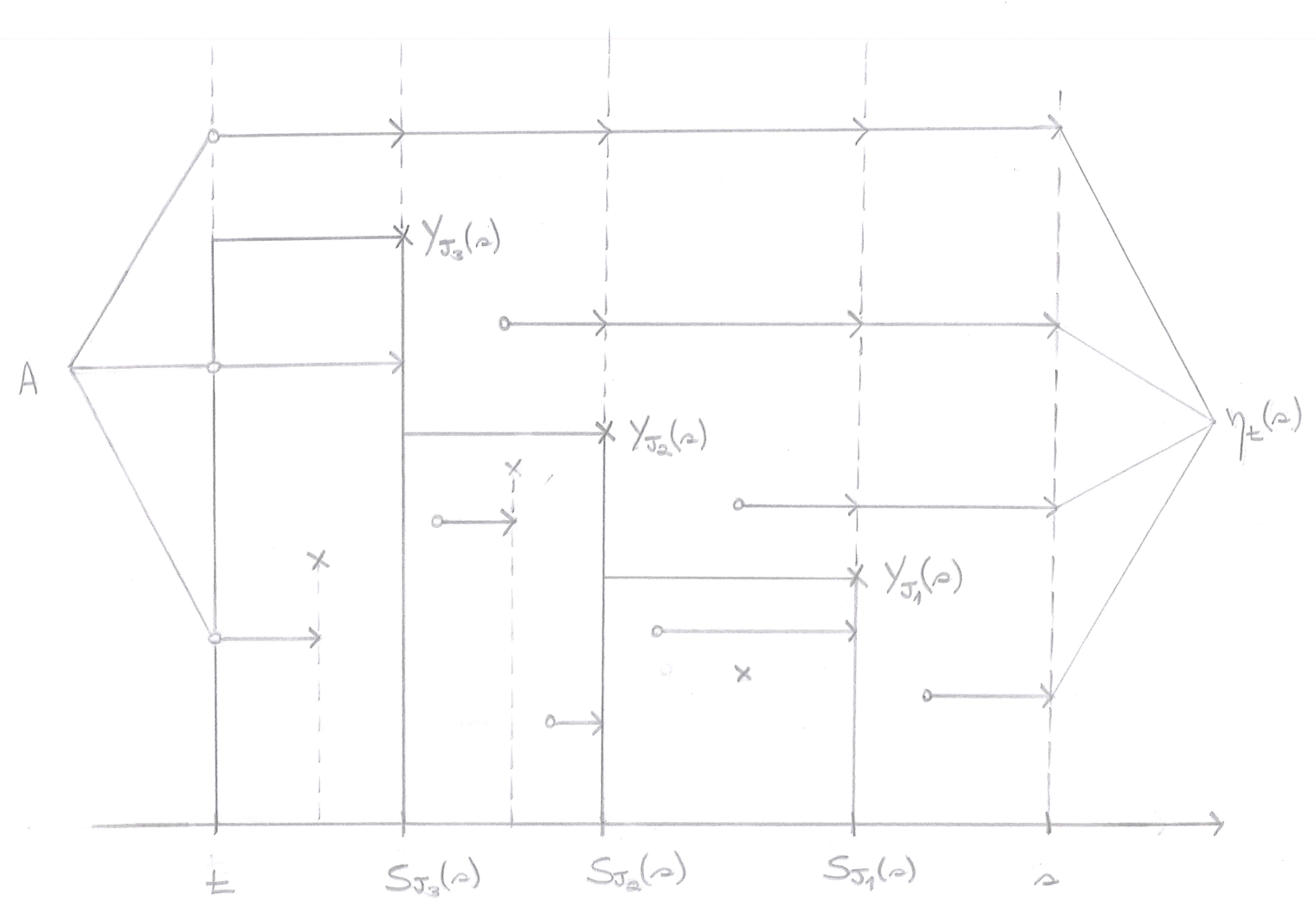

Figura 2.1: Possível realização do processo $\eta_{t}$ no intervalo $[t, s]$

Assim, $\left\{\eta_{t}: t \in \mathbb{R}\right\}$ é uma família de processos estocásticos. Para cada $t \in \mathbb{R}, \eta_{t}=\left\{\eta_{t}(s), s \geq t\right\}$ é um processo Markoviano com transições estacionárias tomando valores no sistema de subconjuntos localmente finitos de $\mathbb{R}^{+}$. Logo, para cada $s>t, \eta_{t}(s)$ é um processo pontual em $\mathbb{R}^{+}$. Em particular, temos interesse no processo $\eta_{0}(\cdot)$. Para mais informações sobre processos pontuais, veja Last e Penrose (2017).

\subsection{Resultados}

Nesta seção, enunciamos os principais resultados desta dissertação.

Defina as funções $R_{\star}, R_{\dagger}: \mathbb{R}^{+} \rightarrow \mathbb{R}^{+}$por:

$$
\begin{aligned}
& R_{\star}(x) \doteq-\log \overline{F_{\star}}(x) \\
& R_{\dagger}(x) \doteq-\log \overline{F_{\dagger}}(x)
\end{aligned}
$$

Dizemos que $\eta_{0}$ é transitório se $\left\{s \geq 0: \eta_{0}(s)=\varnothing\right\}$ é limitado quase certamente.

Dizemos que $\eta_{0}$ é recorrente se $\left\{s \geq 0: \eta_{0}(s)=\varnothing\right\}$ é ilimitado quase certamente.

Teorema 1. O processo $\eta_{0}$ é transitório se:

$$
\int_{[0,+\infty)} \frac{\overline{F_{\dagger}}(x)}{\overline{F_{\star}}(x)} R_{\star}(d x)<+\infty
$$

O processo $\eta_{0}$ é recorrente se:

$$
\int_{[0,+\infty)} \frac{\overline{F_{\dagger}}(x)}{\overline{F_{\star}}(x)} R_{\star}(d x)=+\infty
$$


Teorema 2. O processo $\eta_{0}(t)$ converge em distribuição para $\hat{\eta}$ quando $t \rightarrow \infty$, onde $\hat{\eta}$ é dado por:

$$
\hat{\eta} \doteq\left\{X_{i}: T_{i} \in\left(S_{J_{1}}(0), 0\right]\right\} \cup\left(\bigcup_{k \geq 1}\left\{X_{i}>Y_{J_{k}}(0): T_{i} \in\left(S_{J_{k+1}}(0), S_{J_{k}}(0)\right]\right\}\right)
$$

Teorema 3. O número de espécies na distribuição limite \#̂́ é finito se:

$$
\int_{[0,+\infty)} \frac{\overline{F_{\star}}(x)}{\overline{F_{\dagger}}(x)} R_{\dagger}(d x)<+\infty
$$

O número de espécies na distribuição limite \#̂े é infinito se:

$$
\int_{[0,+\infty)} \frac{\overline{F_{\star}}(x)}{\overline{F_{\dagger}}(x)} R_{\dagger}(d x)=+\infty
$$

Observação 1. Note que o critério para recorrência/transitoriedade é idêntico ao critério para in/finitude da distribuição limite com os papéis de estrela e cruz trocados. Isto ocorre por que as duas questões têm estruturas similares, como veremos mais adiante. 


\section{Capítulo 3}

\section{Recorrência e Transitoriedade}

Neste capítulo, analisamos a recorrência/transitoriedade do processo $\eta_{0}$, como enunciada na Seção 2.2. Na Seção 3.1, construímos uma escada de recordes usando o processo de surgimentos e as aptidões associadas. Na Seção 3.2, calculamos a transformada de Laplace do número de extinções acima da escada de aptidões recordes, via funcional de Laplace. Na Seção 3.3, apresentamos critérios equivalentes para a finitude quase-certa da mesma variável aleatória. Na Seção 3.4, demonstramos o Teorema 1. Por fim, na Seção 3.5, apresentamos um exemplo usando a distribuição exponencial. Vamos utilizar alguns resultados que podem ser encontrados no apêndice.

\subsection{Escada de aptidões recordes}

Nesta seção, usamos o processo de surgimentos e as aptidões associadas para construir uma escada de recordes. Defina os índices recordes $I_{k}$ e valores recordes $X_{I_{k}}$ por:

$$
\begin{aligned}
I_{1} & \doteq 1 \\
I_{k+1} & \doteq \min \left\{i>I_{k}: X_{i}>X_{I_{k}}\right\} \quad \text { para cada } \quad k \geq 1
\end{aligned}
$$

Proposição 1. Os pontos $\left(X_{I_{k}}\right)_{k \geq 1}$ formam um processo de Poisson em $\mathbb{R}^{+}$com intensidade $\int_{B} R_{\star}(d x)$, onde $B \in \mathcal{B}\left(\mathbb{R}^{+}\right)$.

A demonstração pode ser encontrada em Resnick (1973). Também incluímos uma demonstração no apêndice.

Denotamos por $T_{I_{k}}$ o instante do k-ésimo recorde e por $\Delta T_{I_{k}} \doteq T_{I_{k+1}}-T_{I_{k}}$ o intervalo entre dois recordes consecutivos.

Proposição 2. Os pontos $\left(X_{I_{k}}, \Delta T_{I_{k}}\right)_{k \geq 1}$ formam um processo de Poisson em $[0,+\infty)^{2}$ com intensidade:

$$
\hat{\mu}_{\star}(C) \doteq \iint_{C} \lambda_{\star} \overline{F_{\star}}(x) e^{-\lambda_{\star} \overline{F_{\star}}(x) s} d s R_{\star}(d x) \quad \text { para cada } \quad C \in \mathcal{B}\left([0,+\infty)^{2}\right)
$$

Demonstração. Condicional a $\left\{\left(X_{I_{k}}\right)_{k \geq 1}=\left(x_{k}\right)_{k \geq 1}\right\}$, as variáveis aleatórias $\Delta T_{I_{k}}$ são independentes entre si e têm distribuição exponencial de taxa $\lambda_{\star} \bar{F}_{\star}\left(x_{k}\right)$. Denote por $K_{\star}(x, B)$ o seguinte kernel de probabilidades, cuja definição pode ser encontrada na Seção A.2:

$$
K_{\star}(x,(0, s]) \doteq 1-e^{-\lambda_{\star} \overline{F_{\star}}(x) s} \quad \text { para cada } \quad(x, s) \in[0,+\infty)^{2}
$$

A extensão do kernel para intervalos é dada por:

$$
K_{\star}\left(x,\left(s_{1}, s_{2}\right]\right) \doteq K_{\star}\left(x,\left(0, s_{2}\right]\right)-K_{\star}\left(x,\left(0, s_{1}\right]\right) \quad \text { para cada } \quad x \in \mathbb{R}^{+},\left(s_{1}, s_{2}\right] \subset \mathbb{R}^{+}
$$

A extensão do kernel para borelianos $B \in \mathcal{B}\left(\mathbb{R}^{+}\right)$é feita decompondo-os em uniões enumeráveis de sequências disjuntas de intervalos, da mesma forma que para medidas induzidas. Os pontos 
$\left(X_{I_{k}}, \Delta T_{I_{k}}\right)_{k \geq 1}$ formam uma $K_{\star}$-marcação do processo de Poisson descrito na Proposição 1 . O resultado segue do Teorema das Marcas, enunciado na Seção A.2.

Defina as regiões aleatórias acima de cada degrau $\left(D_{k}\right)_{k \geq 1}$ e da escada toda $D$ por:

$$
\begin{aligned}
D_{k} & \doteq\left[T_{I_{k}}, T_{I_{k+1}}\right) \times\left[X_{I_{k}},+\infty\right) \quad \text { para } \quad k \geq 1 \\
D & \doteq \bigcup_{k \geq 1} D_{k}
\end{aligned}
$$

A Figura 3.1 mostra uma possível realização dos processos, com a escada de aptidões recordes e alguns eventos de extinção.

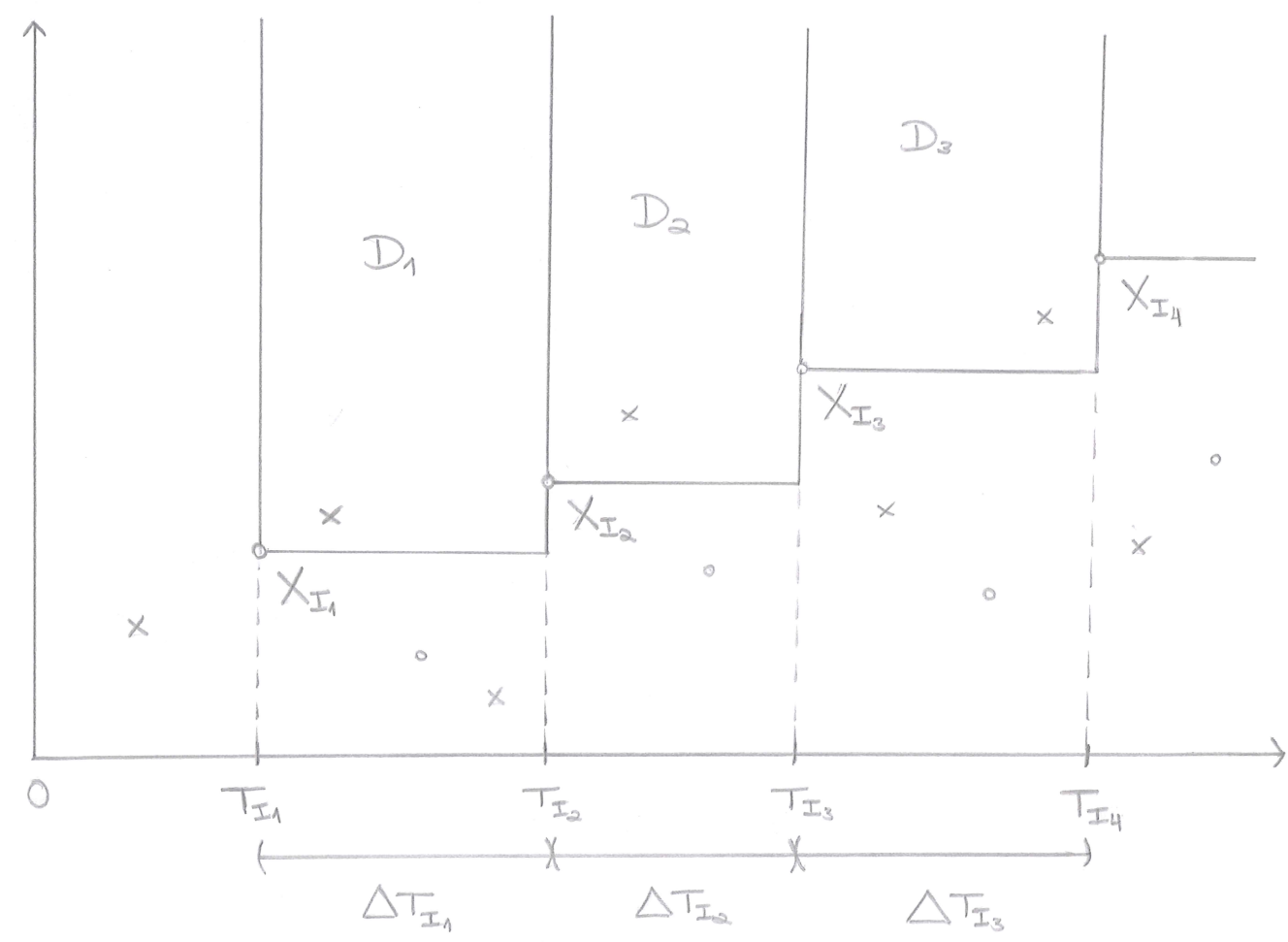

Figura 3.1: Possível realização da escada de aptidões recordes com alguns eventos de extinção

\subsection{Extinções acima da escada via funcional de Laplace}

Nesta seção, utizamos a caracterização de processos de Poisson pelo funcional de Laplace, enunciada na Seção A.2, para encontrar a transformada de Laplace do número de extinções acima da escada de aptidões recordes.

Proposição 3. Os pontos $\left(S_{j}, Y_{j}\right)_{j \geq 1}$ formam um processo de Poisson em $[0,+\infty)^{2}$ denotado por $\hat{\Pi}_{\dagger}$ com intensidade:

$$
\mu_{\dagger}(C) \doteq \iint_{C} \lambda_{\dagger} d t F_{\dagger}(d x) \quad \text { para cada } \quad C \in \mathcal{B}\left([0,+\infty)^{2}\right)
$$

Demonstração. As variáveis aleatórias $\left(Y_{j}\right)_{j \geq 1}$ são independentes entre si e independentes de $\left(S_{j}\right)_{j \geq 1}$. Logo, os pontos $\left(S_{j}, Y_{j}\right)_{j \geq 1}$ formam uma $\mathbb{Q}_{\dagger}$-marcação independente de $\left(S_{j}\right)_{j \geq 1}$, cuja definição pode 
ser encontrada na Seção A.2, onde $\mathbb{Q}_{\dagger}$ é a medida induzida por $Y$. O resultado segue do Teorema das Marcas, enunciado na Seção A.2.

Defina as variáveis aleatórias $\Lambda$ e $M$ por:

$$
\begin{aligned}
\Lambda & \doteq \mu_{\dagger}(D) \\
M & \doteq \#\left(D \cap \hat{\Pi}_{\dagger}\right)
\end{aligned}
$$

Observe que $M$ é o número de eventos de extinção acima da escada de aptidões recordes e que tem distribuição de Poisson com parâmetro $\Lambda$. Logo, $M$ tem distribuição misturada.

Proposição 4. A tranformada de Laplace de $M$ pode ser escrita na forma:

$$
\mathbb{E}\left[e^{-t M}\right]=\mathbb{E}\left[e^{-\left(1-e^{-t}\right) \Lambda}\right]
$$

Demonstração. Como $\hat{\Pi}_{\dagger}$ é processo de Poisson, temos:

$$
\mathbb{P}(M=m \mid \Lambda=\mu)=\frac{\mu^{m}}{m !} e^{-\mu}
$$

Daí segue que:

$$
\mathbb{E}\left[e^{-t M} \mid \Lambda=\mu\right]=\sum_{m \geq 0} e^{-t m} \frac{\mu^{m}}{m !} e^{-\mu}=e^{-\mu} \sum_{m \geq 0} \frac{\left(\mu e^{-t}\right)^{m}}{m !}=e^{-\mu} e^{\mu e^{-t}}=e^{-\left(1-e^{-t}\right) \mu}
$$

A esperança condicional tem a forma:

$$
\mathbb{E}\left[e^{-t M} \mid \Lambda\right]=e^{-\left(1-e^{-t}\right) \Lambda}
$$

Logo:

$$
\mathbb{E}\left[e^{-t M}\right]=\mathbb{E}\left[\mathbb{E}\left[e^{-t M} \mid \Lambda\right]\right]=\mathbb{E}\left[e^{-\left(1-e^{-t}\right) \Lambda}\right]
$$

Defina a função $h_{\dagger}:[0,+\infty)^{2} \rightarrow \mathbb{R}^{+}$por:

$$
h_{\dagger}(x, s) \doteq \lambda_{\dagger} s \overline{F_{\dagger}}(x)
$$

Segue da definição de $\mu_{\dagger}$ na Proposição 3 e da $\sigma$-aditividade da mesma medida, pontualmente, que a variável aleatória $\Lambda$ pode ser escrita como uma soma:

$$
\Lambda=\mu_{\dagger}(D)=\sum_{k \geq 1} \mu_{\dagger}\left(D_{k}\right)=\sum_{k \geq 1} \lambda_{\dagger} \Delta T_{I_{k}} \overline{F_{\dagger}}\left(X_{I_{k}}\right)=\sum_{k \geq 1} h_{\dagger}\left(X_{I_{k}}, \Delta T_{I_{k}}\right)
$$

Proposição 5. A transformada de Laplace de $M$ é:

$$
\mathbb{E}\left[e^{-t M}\right]=\exp \left[-\int_{[0,+\infty)} \frac{\left(1-e^{-t}\right) \lambda_{\dagger} \overline{F_{\dagger}}(x)}{\lambda_{\star} \overline{F_{\star}}(x)+\left(1-e^{-t}\right) \lambda_{\dagger} \overline{F_{\dagger}}(x)} R_{\star}(d x)\right]
$$

Demonstração. Pela caracterização de processos de Poisson por funcional de Laplace, enunciada na 
Seção A.2, temos que:

$$
\begin{aligned}
\mathbb{E}\left[e^{-\left(1-e^{-t}\right) \Lambda}\right] & =\mathbb{E}\left[e^{-\sum_{k \geq 1}\left(1-e^{-t}\right) h_{\dagger}\left(X_{I_{k}}, \Delta T_{I_{k}}\right)}\right] \\
& =\exp \left[-\iint_{[0,+\infty)^{2}}\left(1-e^{-\left(1-e^{-t}\right) h_{\dagger}(x, s)}\right) \hat{\mu}_{\star}(d x, d s)\right] \\
& =\exp \left[-\iint_{[0,+\infty)^{2}}\left(1-e^{-\left(1-e^{-t}\right) \lambda_{\dagger} s \overline{F_{\dagger}}(x)}\right) \lambda_{\star} \overline{F_{\star}}(x) e^{-\lambda_{\star} \overline{F_{\star}}(x) s} d s R_{\star}(d x)\right]
\end{aligned}
$$

Integrando em $s$ obtemos a seguinte expressão:

$$
\begin{aligned}
\mathbb{E}\left[e^{-\left(1-e^{-t}\right) \Lambda}\right] & =\exp \left[-\int_{[0,+\infty)}\left(1-\frac{\lambda_{\star} \overline{F_{\star}}(x)}{\lambda_{\star} \overline{F_{\star}}(x)+\left(1-e^{-t}\right) \lambda_{\dagger} \overline{F_{\dagger}}(x)}\right) R_{\star}(d x)\right] \\
& =\exp \left[-\int_{[0,+\infty)} \frac{\left(1-e^{-t}\right) \lambda_{\dagger} \overline{F_{\dagger}}(x)}{\lambda_{\star} \overline{F_{\star}}(x)+\left(1-e^{-t}\right) \lambda_{\dagger} \overline{F_{\dagger}}(x)} R_{\star}(d x)\right]
\end{aligned}
$$

O resultado segue da Proposição 4.

\subsection{Finitude das extinções}

Nesta seção, apresentamos critérios equivalentes para a finitude quase-certa de $M$.

Defina as funções $\phi: \mathbb{R}^{+} \rightarrow \mathbb{R}^{+}$e $\psi:[0,1] \rightarrow[0,1]$ por:

$$
\begin{aligned}
\phi(t) & \doteq \int_{[0,+\infty)} \frac{\left(1-e^{-t}\right) \lambda_{\dagger} \overline{F_{\dagger}}(x)}{\lambda_{\star} \overline{F_{\star}}(x)+\left(1-e^{-t}\right) \lambda_{\dagger} \overline{F_{\dagger}}(x)} R_{\star}(d x) \\
\psi(u) & \doteq \overline{F_{\dagger}} \circ \overline{F_{\star}}-1(u)
\end{aligned}
$$

Denote por $\phi(+\infty) \in[0,+\infty]$ o seguinte limite:

$$
\phi(+\infty) \doteq \lim _{t \rightarrow+\infty} \phi(t)
$$

Pelo Teorema de Convergência Monótona:

$$
\phi(+\infty)=\int_{[0,+\infty)} \frac{\lambda_{\dagger} \overline{F_{\dagger}}(x)}{\lambda_{\star} \overline{F_{\star}}(x)+\lambda_{\dagger} \overline{F_{\dagger}}(x)} R_{\star}(d x)
$$

Vamos calcular $\mathbb{E}[M]$. Por (3.11), temos que:

$$
\mathbb{E}[M]=\mathbb{E}[\mathbb{E}[M \mid \Lambda]]=\mathbb{E}[\Lambda]
$$

Podemos encontrar $\mathbb{E}[\Lambda]$ usando a Fórmula de Campbell, enunciada na Seção A.2:

$$
\begin{aligned}
\mathbb{E}[\Lambda] & =\mathbb{E}\left[\sum_{k \geq 1} h_{\dagger}\left(X_{I_{k}}, \Delta T_{I_{k}}\right)\right]=\iint_{[0,+\infty)} h_{\dagger}(x, s) \hat{\mu}_{\star}(d x, d s) \\
& =\iint_{[0,+\infty)} \lambda_{\dagger} s \overline{F_{\dagger}}(x) \lambda_{\star} \overline{F_{\star}}(x) e^{-\lambda_{\star} \overline{F_{\star}}(x) s} d s \cdot R_{\star}(d x) \\
& =\int_{[0,+\infty)} \lambda_{\dagger} \overline{F_{\dagger}}(x)\left(\int_{[0,+\infty)} s \lambda_{\star} \overline{F_{\star}}(x) e^{-\lambda_{\star} \overline{F_{\star}}(x) s} d s\right) R_{\star}(d x) \\
& =\int_{[0,+\infty)} \frac{\lambda_{\dagger} \overline{F_{\dagger}}(x)}{\lambda_{\star} \overline{F_{\star}}(x)} R_{\star}(d x)
\end{aligned}
$$


onde a integral em $s$ é feita por partes. Assim, temos:

$$
\mathbb{E}[M]=\frac{\lambda_{\dagger}}{\lambda_{\star}} \int_{[0,+\infty)} \frac{\overline{F_{\dagger}}(x)}{\overline{F_{\star}}(x)} R_{\star}(d x)
$$

Proposição 6. São equivalentes:

i) $\phi(+\infty)<+\infty$

ii) $\mathbb{P}(M<+\infty)=1$

iii) $\mathbb{E}[M]<+\infty$

iv) $\frac{\psi(u)}{u^{2}}$ é integrável na origem

v) $\psi\left(s^{-1}\right)$ é integrável no infinito

Demonstração. Vamos começar mostrando que (i) $\Rightarrow($ ii). Note que:

$$
0 \leq\left(1-e^{-t}\right) \frac{\lambda_{\dagger} \overline{F_{\dagger}}(x)}{\lambda_{\star} \overline{F_{\star}}(x)+\lambda_{\dagger} \overline{F_{\dagger}}(x)} \leq \frac{\left(1-e^{-t}\right) \lambda_{\dagger} \overline{F_{\dagger}}(x)}{\lambda_{\star} \overline{F_{\star}}(x)+\left(1-e^{-t}\right) \lambda_{\dagger} \overline{F_{\dagger}}(x)} \leq \frac{\lambda_{\dagger} \overline{F_{\dagger}}(x)}{\lambda_{\star} \overline{F_{\star}}(x)+\lambda_{\dagger} \overline{F_{\dagger}}(x)}
$$

Integrando com respeito a $R_{\star}(d x)$ temos:

$$
\left(1-e^{-t}\right) \phi(+\infty) \leq \phi(t) \leq \phi(+\infty)
$$

Se $\phi(+\infty)<+\infty$, pelo Teorema da Convergência Dominada, temos $\lim _{t \downarrow 0} \phi(t)=0$. Logo:

$$
\lim _{t \downarrow 0} \mathbb{E}\left[e^{-t M}\right]=\lim _{t \downarrow 0} \exp [-\phi(t)]=\exp \left[-\lim _{t \downarrow 0} \phi(t)\right]=1
$$

Por outro lado:

$$
\lim _{t \downarrow 0} \mathbb{E}\left[e^{-t M}\right]=\lim _{t \downarrow 0} \sum_{m \geq 0} e^{-t m} \mathbb{P}(M=m)+0 \cdot \mathbb{P}(M=+\infty)=\sum_{m \geq 0} \mathbb{P}(M=m)=\mathbb{P}(M<+\infty)
$$

Assim, $\mathbb{P}(M<+\infty)=1$ e temos que (i) $\Rightarrow$ (ii).

Agora vamos ver que (ii) $\Rightarrow$ (i). Isto será mostrado por contradição.

Suponha que $\phi(+\infty)=+\infty$. Por (3.28), temos $\phi(t)=+\infty$ para cada $t>0$. Daí segue:

$$
\begin{aligned}
& \mathbb{E}\left[e^{-t M}\right]=\exp [-\phi(t)]=0 \text { para cada } t>0 \\
\Rightarrow & \mathbb{P}\left(e^{-t M}=0\right)=1 \text { para cada } t>0 \\
\Rightarrow & \mathbb{P}(M=+\infty)=1
\end{aligned}
$$

Por contradição, temos que (ii) $\Rightarrow$ (i).

Em seguida, vamos mostrar que (iii) $\Rightarrow($ i). Note que:

$$
\frac{\lambda_{\dagger} \overline{F_{\dagger}}(x)}{\lambda_{\star} \overline{F_{\star}}(x)+\lambda_{\dagger} \overline{F_{\dagger}}(x)} \leq \frac{\lambda_{\dagger} \overline{F_{\dagger}}(x)}{\lambda_{\star} \overline{F_{\star}}(x)}
$$

Integrando com respeito a $R_{\star}(d x)$ temos:

$$
\phi(+\infty) \leq \mathbb{E}[M]
$$

Se $\mathbb{E}[M]<+\infty$, então $\phi(+\infty)<+\infty$ e temos que (iii) $\Rightarrow$ (i). 
Para mostrar que $(\mathrm{i}) \Rightarrow($ iii), vamos fazer a seguinte troca de variáveis:

$$
\begin{aligned}
& y=R_{\star}(x)=-\log \overline{F_{\star}}(x) \Rightarrow e^{-y}=\overline{F_{\star}}(x) \Rightarrow x=\overline{F_{\star}}-1\left(e^{-y}\right) \\
& \overline{F_{\dagger}}(x)=\overline{F_{\dagger}} \circ \overline{F_{\star}}-1\left(e^{-y}\right)=\psi\left(e^{-y}\right) \\
& u=e^{-y} \Rightarrow d y=-\frac{d u}{u}
\end{aligned}
$$

Assim, podemos reescrever a integral na forma:

$$
\begin{aligned}
\phi(+\infty) & =\int_{[0,+\infty)} \frac{\lambda_{\dagger} \overline{F_{\dagger}}(x)}{\lambda_{\star} \overline{F_{\star}}(x)+\lambda_{\dagger} \overline{F_{\dagger}}(x)} R_{\star}(d x) \\
& =\int_{[0,+\infty)} \frac{\lambda_{\dagger} \psi\left(e^{-y}\right)}{\lambda_{\star} e^{-y}+\lambda_{\dagger} \psi\left(e^{-y}\right)} d y=\int_{0}^{1} \frac{\lambda_{\dagger} \psi(u)}{\lambda_{\star} u+\lambda_{\dagger} \psi(u)} \frac{d u}{u}
\end{aligned}
$$

Note que:

$$
0 \leq \frac{\lambda_{\dagger} \psi(u)}{\left(\lambda_{\star} u+\lambda_{\dagger} \psi(u)\right) u} I_{(\varepsilon, 2 \varepsilon)}(u) \leq \frac{\lambda_{\dagger} \psi(u)}{\left(\lambda_{\star} u+\lambda_{\dagger} \psi(u)\right) u} \quad \text { para cada } \quad \varepsilon \in\left(0, \frac{1}{2}\right)
$$

Se $\phi(+\infty)<+\infty$, pelo Teorema de Convergência Dominada, temos:

$$
\lim _{\varepsilon \downarrow 0} \int_{\varepsilon}^{2 \varepsilon} \frac{\lambda_{\dagger} \psi(u)}{\left(\lambda_{\star} u+\lambda_{\dagger} \psi(u)\right) u} d u=\lim _{\varepsilon \downarrow 0} \int_{0}^{1} \frac{\lambda_{\dagger} \psi(u)}{\left(\lambda_{\star} u+\lambda_{\dagger} \psi(u)\right) u} I_{(\varepsilon, 2 \varepsilon)}(u) d u=0
$$

Como:

$$
0 \leq \frac{\lambda_{\dagger} \psi(\varepsilon)}{\lambda_{\star} u+\lambda_{\dagger} \psi(\varepsilon)} \leq \frac{\lambda_{\dagger} \psi(u)}{\lambda_{\star} u+\lambda_{\dagger} \psi(u)} \quad \text { para cada } \quad u \in(\varepsilon, 1)
$$

Temos que:

$$
0 \leq \int_{\varepsilon}^{2 \varepsilon} \frac{\lambda_{\dagger} \psi(\varepsilon)}{\left(\lambda_{\star} u+\lambda_{\dagger} \psi(\varepsilon)\right) u} d u \leq \int_{\varepsilon}^{2 \varepsilon} \frac{\lambda_{\dagger} \psi(u)}{\left(\lambda_{\star} u+\lambda_{\dagger} \psi(u)\right) u} d u
$$

Logo:

$$
\lim _{\varepsilon \downarrow 0} \int_{\varepsilon}^{2 \varepsilon} \frac{\lambda_{\dagger} \psi(\varepsilon)}{\left(\lambda_{\star} u+\lambda_{\dagger} \psi(\varepsilon)\right) u} d u=0
$$

Resolvendo a integral:

$$
\begin{aligned}
\int_{\varepsilon}^{2 \varepsilon} \frac{\lambda_{\dagger} \psi(\varepsilon)}{\left(\lambda_{\star} u+\lambda_{\dagger} \psi(\varepsilon)\right) u} d u & =\int_{\varepsilon}^{2 \varepsilon} \frac{1}{u} d u-\int_{\varepsilon}^{2 \varepsilon} \frac{\lambda_{\star}}{\lambda_{\star} u+\lambda_{\dagger} \psi(\varepsilon)} d u \\
& =\left.\log u\right|_{\varepsilon} ^{2 \varepsilon}-\left.\log \left(\lambda_{\star} u+\lambda_{\dagger} \psi(\varepsilon)\right)\right|_{\varepsilon} ^{2 \varepsilon} \\
& =\log \frac{2\left(\lambda_{\star} \varepsilon+\lambda_{\dagger} \psi(\varepsilon)\right)}{\lambda_{\star} 2 \varepsilon+\lambda_{\dagger} \psi(\varepsilon)} \\
& =\log \left(1+\frac{\lambda_{\dagger} \psi(\varepsilon)}{\lambda_{\star} 2 \varepsilon+\lambda_{\dagger} \psi(\varepsilon)}\right)
\end{aligned}
$$

Por (3.41) temos:

$$
\begin{aligned}
\lim _{\varepsilon \downarrow 0} \log \left(1+\frac{\lambda_{\dagger} \psi(\varepsilon)}{\lambda_{\star} 2 \varepsilon+\lambda_{\dagger} \psi(\varepsilon)}\right) & =0 \\
\Rightarrow \lim _{\varepsilon \downarrow 0} \frac{\lambda_{\dagger} \psi(\varepsilon)}{\lambda_{\star} 2 \varepsilon+\lambda_{\dagger} \psi(\varepsilon)} & =0 \\
\Rightarrow \lim _{\varepsilon \downarrow 0} \frac{\lambda_{\dagger} \psi(\varepsilon)}{\lambda_{\star} \varepsilon} & =0
\end{aligned}
$$


Já que:

$$
\frac{\lambda_{\dagger} \psi(\varepsilon)}{\lambda_{\star} 2 \varepsilon+\lambda_{\dagger} \psi(\varepsilon)} \rightarrow 0 \Rightarrow \frac{\lambda_{\star} 2 \varepsilon+\lambda_{\dagger} \psi(\varepsilon)}{\lambda_{\dagger} \psi(\varepsilon)} \rightarrow+\infty \Rightarrow \frac{\lambda_{\star} 2 \varepsilon}{\lambda_{\dagger} \psi(\varepsilon)} \rightarrow+\infty \Rightarrow \frac{\lambda_{\dagger} \psi(\varepsilon)}{\lambda_{\star} \varepsilon} \rightarrow 0
$$

Por (3.44), existe $\delta \in(0,1)$ tal que $\lambda_{\dagger} \psi(u) \leq \lambda_{\star} u$ para cada $u \in(0, \delta)$. Daí segue que:

$\int_{0}^{\delta} \frac{\lambda_{\dagger} \psi(u)}{\lambda_{\star} u} \frac{d u}{u}=2 \int_{0}^{\delta} \frac{\lambda_{\dagger} \psi(u)}{\lambda_{\star} u+\lambda_{\star} u} \frac{d u}{u} \leq 2 \int_{0}^{\delta} \frac{\lambda_{\dagger} \psi(u)}{\lambda_{\star} u+\lambda_{\dagger} \psi(u)} \frac{d u}{u} \leq 2 \int_{0}^{1} \frac{\lambda_{\dagger} \psi(u)}{\lambda_{\star} u+\lambda_{\dagger} \psi(u)} \frac{d u}{u}$

Logo:

$$
\frac{\lambda_{\dagger}}{\lambda_{\star}} \int_{0}^{\delta} \frac{\psi(u)}{u^{2}} d u<+\infty
$$

Por outro lado, como $\psi(u) \leq 1$ e $\delta^{2} \leq u^{2}$ para cada $u \in(\delta, 1)$, temos:

$$
\frac{\lambda_{\dagger}}{\lambda_{\star}} \int_{\delta}^{1} \frac{\psi(u)}{u^{2}} d u \leq \frac{\lambda_{\dagger}}{\lambda_{\star}} \int_{\delta}^{1} \frac{1}{\delta^{2}} d u=\frac{\lambda_{\dagger}(1-\delta)}{\lambda_{\star} \delta^{2}}
$$

Assim:

$$
\frac{\lambda_{\dagger}}{\lambda_{\star}} \int_{0}^{1} \frac{\psi(u)}{u^{2}} d u=\frac{\lambda_{\dagger}}{\lambda_{\star}} \int_{0}^{\delta} \frac{\psi(u)}{u^{2}} d u+\frac{\lambda_{\dagger}}{\lambda_{\star}} \int_{\delta}^{1} \frac{\psi(u)}{u^{2}} d u<+\infty
$$

Reescrevendo a expressão para $\mathbb{E}[M]$ em termos de $\psi$, temos que:

$$
\mathbb{E}[M]=\frac{\lambda_{\dagger}}{\lambda_{\star}} \int_{[0,+\infty)} \frac{\overline{F_{\dagger}}(x)}{\overline{F_{\star}}(x)} R_{\star}(d x)=\frac{\lambda_{\dagger}}{\lambda_{\star}} \int_{[0,+\infty)} \frac{\psi\left(e^{-y}\right)}{e^{-y}} d y=\frac{\lambda_{\dagger}}{\lambda_{\star}} \int_{0}^{1} \frac{\psi(u)}{u^{2}} d u<+\infty
$$

Portanto, temos que (i) $\Rightarrow$ (iii).

Para ver que (iii) $\Leftrightarrow($ iv), note que:

$$
\int_{\delta}^{1} \frac{\psi(u)}{u^{2}} d u<+\infty \quad \text { para cada } \delta \in(0,1)
$$

Como:

$$
\mathbb{E}[M]=\frac{\lambda_{\dagger}}{\lambda_{\star}} \int_{0}^{\delta} \frac{\psi(u)}{u^{2}} d u+\frac{\lambda_{\dagger}}{\lambda_{\star}} \int_{\delta}^{1} \frac{\psi(u)}{u^{2}} d u
$$

Temos que:

$$
\mathbb{E}[M]<+\infty \Leftrightarrow \int_{0}^{\delta} \frac{\psi(u)}{u^{2}} d u<+\infty \quad \text { para algum } \quad \delta \in(0,1)
$$

Logo, (iii) $\Leftrightarrow$ (iv).

Por fim, vamos mostrar que (iv) $\Leftrightarrow(v)$. Fazendo a seguinte substituição:

$$
s=u^{-1} \Rightarrow \frac{d s}{d u}=-u^{-2} \Rightarrow d s=-\frac{d u}{u^{2}}
$$

podemos reescrever a expressão para $\mathbb{E}[M]$ como:

$$
\mathbb{E}[M]=-\frac{\lambda_{\dagger}}{\lambda_{\star}} \int_{+\infty}^{1} \psi\left(s^{-1}\right) d s=\frac{\lambda_{\dagger}}{\lambda_{\star}} \int_{1}^{+\infty} \psi\left(s^{-1}\right) d s
$$

Note que:

$$
\int_{1}^{\delta} \psi\left(s^{-1}\right) d s<+\infty \quad \text { para cada } \quad \delta \in \mathbb{R}^{+}
$$


Logo:

$$
\mathbb{E}[M]<+\infty \Leftrightarrow \int_{\delta}^{+\infty} \psi\left(s^{-1}\right) d s<+\infty \quad \text { para algum } \quad \delta \in \mathbb{R}^{+}
$$

Assim, (iv) $\Leftrightarrow(\mathrm{v})$.

Observação 2. A expressão para $\phi(t)$ pode ser reescrita em termos de $\psi(u)$ fazendo a mesma troca de variáveis que levou a (3.36):

$$
\phi(t)=\int_{[0,+\infty)} \frac{\left(1-e^{-t}\right) \lambda_{\dagger} \overline{F_{\dagger}}(x)}{\lambda_{\star} \overline{F_{\star}}(x)+\left(1-e^{-t}\right) \lambda_{\dagger} \overline{F_{\dagger}}(x)} R_{\star}(d x)=\int_{0}^{1} \frac{\left(1-e^{-t}\right) \lambda_{\dagger} \psi(u)}{\lambda_{\star} u+\left(1-e^{-t}\right) \lambda_{\dagger} \psi(u)} \frac{d u}{u}
$$

Logo, a distribuição de $M$ depende somente de $\psi(u)$.

\subsection{Demonstração do Teorema 1}

Nesta seção, apresentamos a demonstração do Teorema 1, enunciado na Seção 2.2. Em palavras, a demonstração segue o seguinte raciocínio: satisfeita a condição de finitude quase-certa de $M$, sabemos que existe um último evento de extinção acima da escada de aptidões recordes; a partir do surgimento da aptidão recorde seguinte, sempre haverá ao menos uma espécie no sistema. Satisfeita a condição de infinitude quase-certa de $M$, fixamos arbitrariamente um instante de referência e podemos encontrar eventos de extinção acima da escada de aptidões recordes posteriores a este instante.

Demonstração. Pela Proposição 6, se $\int_{[0,+\infty)} \frac{\overline{F_{\dagger}}(x)}{\overline{F_{\star}}(x)} R_{\star}(d x)<+\infty$, então:

$$
M=\#\left(D \cap \hat{\Pi}_{\dagger}\right)<+\infty \quad \text { quase certamente }
$$

Dado $\{M<+\infty\}$, considere o índice do último evento de extinção em $D$ :

$$
\hat{j} \doteq \max \left\{j:\left(S_{j}, Y_{j}\right) \in D\right\}
$$

e note que existe um único índice $k \geq 1$ tal que $\left(S_{\hat{j}}, Y_{\hat{j}}\right) \in D_{k}$. Vamos denotá-lo por $\hat{k}$.

A próxima aptidão recorde surge no instante $T_{I_{\hat{k}+1}}$, logo:

$$
\eta_{0}(s) \neq \varnothing \quad \text { para cada } \quad s \geq T_{I_{\hat{k}+1}}
$$

já que:

$$
D \cap \hat{\Pi}_{\dagger} \cap\left[T_{I_{\hat{k}+1}},+\infty\right) \times[0,+\infty)=\varnothing
$$

Assim:

$$
\left\{s \geq 0: \eta_{0}(s)=\varnothing\right\} \subset\left[0, T_{I_{\hat{k}+1}}\right)
$$

Daí segue que $\left\{s \geq 0: \eta_{0}(s)=\varnothing\right\}$ é limitado quase certamente e o processo $\eta_{0}$ é transitório.

Pela Proposição 6, se $\int_{[0,+\infty)} \frac{\overline{F_{\dagger}}(x)}{\overline{F_{\star}}(x)} R_{\star}(d x)=+\infty$, então:

$$
M=\#\left(D \cap \hat{\Pi}_{\dagger}\right)=+\infty \quad \text { quase certamente }
$$

Dado $\{M=+\infty\}$, suponha, por absurdo, que existe $\hat{s}>0$ tal que:

$$
\left\{s \geq 0: \eta_{0}(s)=\varnothing\right\} \subset[0, \hat{s}]
$$


Como $\mu_{\dagger}([0, \hat{s}) \times[0,+\infty))=\lambda_{\dagger} \hat{s}<+\infty$ temos que:

$$
\#\left([0, \hat{s}) \times[0,+\infty) \cap \hat{\Pi}_{\dagger}\right)<+\infty \quad \text { quase certamente }
$$

Daí segue que:

$$
\#\left([0, \hat{s}) \times[0,+\infty) \cap D \cap \hat{\Pi}_{\dagger}\right)<+\infty \quad \text { quase certamente }
$$

Logo:

$$
\#\left([\hat{s},+\infty) \times[0,+\infty) \cap D \cap \hat{\Pi}_{\dagger}\right)=+\infty \text { quase certamente }
$$

Basta escolher um evento de extinção $\left(S_{j}, Y_{j}\right) \in[\hat{s},+\infty) \times[0,+\infty) \cap D$ e ver que:

$$
\eta_{0}\left(S_{j}\right)=\varnothing
$$

Assim, temos uma contradição e $\left\{s \geq 0: \eta_{0}(s)=\varnothing\right\}$ é ilimitado quase certamente. Neste caso, o processo $\eta_{0}$ é recorrente.

\subsection{Exemplo}

Nesta seção, apresentamos um exemplo usando a distribuição exponencial. Vamos supor que:

$$
\begin{aligned}
& \overline{F_{\star}}(x)=e^{-\alpha_{\star} x} \mathbf{1}_{[0,+\infty)}(x) \\
& \overline{F_{\dagger}}(x)=e^{-\alpha_{\dagger} x} \mathbf{1}_{[0,+\infty)}(x)
\end{aligned}
$$

$\operatorname{Para} \overline{F_{\star}}(x)=e^{-\alpha_{\star} x}$, temos:

$$
R_{\star}(d x)=\frac{d}{d x}\left(-\log \overline{F_{\star}}(x)\right)=\frac{f_{\star}(x)}{\overline{F_{\star}}(x)} d x=\alpha_{\star} d x
$$

Avaliando a integral:

$$
\int_{0}^{+\infty} \frac{\overline{F_{\dagger}}(x)}{\overline{F_{\star}}(x)} R_{\star}(d x)=\alpha_{\star} \int_{0}^{+\infty} e^{-\left(\alpha_{\dagger}-\alpha_{\star}\right) x} d x=\left\{\begin{array}{lll}
\frac{\alpha_{\star}}{\alpha_{\dagger}-\alpha_{\star}}, & \text { se } & \alpha_{\dagger}>\alpha_{\star} \\
+\infty, & \text { se } & \alpha_{\dagger} \leq \alpha_{\star}
\end{array}\right.
$$

Para $\alpha_{\dagger} \leq \alpha_{\star}$, temos $M=+\infty$ quase certamente, pela Proposição 6. Neste caso, o processo $\eta_{0}$ é recorrente, pelo Teorema 1 .

Para $\alpha_{\dagger}>\alpha_{\star}$, podemos encontrar a distribuição de $\Lambda$ e $M$ resolvendo a seguinte integral:

$$
\begin{aligned}
\int_{0}^{+\infty} \frac{\lambda_{\dagger}(t) \overline{F_{\dagger}}(x)}{\lambda_{\star} \overline{F_{\star}}(x)+\lambda_{\dagger}(t) \overline{F_{\dagger}}(x)} R_{\star}(d x) & =\int_{0}^{+\infty} \frac{\alpha_{\star} \lambda_{\dagger}(t) e^{-\alpha_{\dagger} x}}{\lambda_{\star} e^{-\alpha_{\star} x}+\lambda_{\dagger}(t) e^{-\alpha_{\dagger} x}} d x \\
& =\int_{0}^{+\infty} \frac{\alpha_{\star} \lambda_{\dagger}(t)}{\lambda_{\star} e^{\left(\alpha_{\dagger}-\alpha_{\star}\right) x}+\lambda_{\dagger}(t)} d x \\
& =\frac{\alpha_{\star}}{\alpha_{\dagger}-\alpha_{\star}} \int_{1}^{+\infty} \frac{\lambda_{\dagger}(t)}{\lambda_{\star} u+\lambda_{\dagger}(t)} \frac{d u}{u} \\
& =\frac{\alpha_{\star}}{\alpha_{\dagger}-\alpha_{\star}}\left(\int_{1}^{+\infty} \frac{d u}{u}-\int_{1}^{+\infty} \frac{\lambda_{\star} d u}{\lambda_{\star} u+\lambda_{\dagger}(t)}\right) \\
& =\frac{\alpha_{\star}}{\alpha_{\dagger}-\alpha_{\star}}\left(\left.\log u\right|_{1} ^{+\infty}-\left.\log \left(\lambda_{\star} u+\lambda_{\dagger}(t)\right)\right|_{1} ^{+\infty}\right) \\
& =\left.\frac{\alpha_{\star}}{\alpha_{\dagger}-\alpha_{\star}} \log \frac{u}{\lambda_{\star} u+\lambda_{\dagger}(t)}\right|_{1} ^{+\infty}
\end{aligned}
$$




$$
\begin{aligned}
& =\left.\frac{\alpha_{\star}}{\alpha_{\dagger}-\alpha_{\star}} \log \frac{e^{\left(\alpha_{\dagger}-\alpha_{\star}\right) x}}{\lambda_{\star} e^{\left(\alpha_{\dagger}-\alpha_{\star}\right) x}+\lambda_{\dagger}(t)}\right|_{0} ^{+\infty} \\
& =\frac{\alpha_{\star}}{\alpha_{\dagger}-\alpha_{\star}}\left(\log \lim _{x \rightarrow+\infty} \frac{e^{\left(\alpha_{\dagger}-\alpha_{\star}\right) x}}{\lambda_{\star} e^{\left(\alpha_{\dagger}-\alpha_{\star}\right) x}+\lambda_{\dagger}(t)}-\log \frac{1}{\lambda_{\star}+\lambda_{\dagger}(t)}\right) \\
& =\frac{\alpha_{\star}}{\alpha_{\dagger}-\alpha_{\star}}\left(\log \frac{1}{\lambda_{\star}}-\log \frac{1}{\lambda_{\star}+\lambda_{\dagger}(t)}\right) \\
& =-\log \left(\frac{\lambda_{\star}}{\lambda_{\star}+\lambda_{\dagger}(t)}\right)^{\frac{\alpha_{\star}}{\alpha_{\dagger}-\alpha_{\star}}}
\end{aligned}
$$

Pela caracterização de processos de Poisson por funcional de Laplace, enunciada na Seção A.2, temos que:

$$
\mathbb{E}\left[e^{-t \Lambda}\right]=\mathbb{E}\left[e^{-\sum_{k \geq 1} t . h_{\dagger}\left(X_{I_{k}}, \Delta T_{I_{k}}\right)}\right]=\exp \left[-\int_{[0,+\infty)} \frac{t \lambda_{\dagger} \overline{F_{\dagger}}(x)}{\lambda_{\star} \overline{F_{\star}}(x)+t \lambda_{\dagger} \overline{F_{\dagger}}(x)} R_{\star}(d x)\right]
$$

Basta tomar $\lambda_{\dagger}(t)=t \lambda_{\dagger}$ na integral anterior para ver que:

$$
\mathbb{E}\left[e^{-t \Lambda}\right]=\left(\frac{\lambda_{\star}}{\lambda_{\star}+\lambda_{\dagger}(t)}\right)^{\frac{\alpha_{\star}}{\alpha_{\dagger}-\alpha_{\star}}}=\left(1+\frac{t}{\frac{\lambda_{\star}}{\lambda_{\dagger}}}\right)^{-\frac{\alpha_{\star}}{\alpha_{\dagger}-\alpha_{\star}}}=\left(1+\frac{t}{\beta}\right)^{-r}
$$

onde $r=\frac{\alpha_{\star}}{\alpha_{\dagger}-\alpha_{\star}}$ e $\beta=\frac{\lambda_{\star}}{\lambda_{\dagger}}$.

Identificamos que $\Lambda$ tem distribuição gama com parâmetros $r$ e $\beta$.

Pela Proposição 5, tomando $\lambda_{\dagger}(t)=\left(1-e^{-t}\right) \lambda_{\dagger}$, obtemos:

$$
\mathbb{E}\left[e^{-t M}\right]=\left(\frac{\lambda_{\star}}{\lambda_{\star}+\lambda_{\dagger}\left(1-e^{-t}\right)}\right)^{\frac{\alpha_{\star}}{\alpha_{\dagger}-\alpha_{\star}}}=\left(\frac{\frac{\lambda_{\star}}{\lambda_{\star}+\lambda_{\dagger}}}{1-\frac{\lambda_{\dagger}}{\lambda_{\star}+\lambda_{\dagger}} e^{-t}}\right)^{\frac{\alpha_{\star}}{\alpha_{\dagger}-\alpha_{\star}}}=\left(\frac{1-p}{1-p e^{-t}}\right)^{r}
$$

onde $r=\frac{\alpha_{\star}}{\alpha_{\dagger}-\alpha_{\star}}$ e $p=\frac{\lambda_{\dagger}}{\lambda_{\star}+\lambda_{\dagger}}$.

Identificamos que $M$ tem distribuição binomial negativa com parâmetros $r$ e $p$. Daí segue que:

$$
\mathbb{P}(M=m)=\left(\begin{array}{c}
m+r-1 \\
m
\end{array}\right) p^{m}(1-p)^{r} \quad, \quad m \in\{0,1,2, \ldots\}
$$

Logo, $M<+\infty$ quase certamente para $\alpha_{\dagger}>\alpha_{\star}$. Este resultado também pode ser obtido diretamente da Proposição 6. Neste caso, o processo é transitório, pelo Teorema 1.

Observação 3. Note que:

$$
\begin{gathered}
{\overline{F_{\star}}}^{-1}(u)=-\log u^{\frac{1}{\alpha_{\star}}} \\
\psi(u)=\overline{F_{\dagger}} \circ \overline{F_{\star}}-1(u)=e^{-\alpha_{\dagger}\left(-\log u^{\frac{1}{\alpha_{\star}}}\right)=u^{\frac{\alpha_{\dagger}}{\alpha_{\star}}}}
\end{gathered}
$$

Como vimos na Observação 2, a distribuição de $M$ depende somente de $\psi(u)$.

Assim, (3.75) vale sempre que:

$$
\overline{F_{\dagger}}(x)=\overline{F_{\star}}(x)^{\frac{\alpha_{\dagger}}{\alpha_{\star}}}
$$

Basta tomar $x={\overline{F_{\star}}}^{-1}(u)$ para ver que:

$$
\psi(u)=\overline{F_{\dagger}} \circ{\overline{F_{\star}}}^{-1}(u)=\left(\overline{F_{\star}} \circ \overline{F_{\star}}-1(u)\right)^{\frac{\alpha_{\dagger}}{\alpha_{\star}}}=u^{\frac{\alpha_{\dagger}}{\alpha_{\star}}}
$$




\section{Capítulo 4}

\section{Distribuição Limite}

Neste capítulo, mostramos a existência da distribuição limite para $\eta_{0}(t)$ quando $t \rightarrow+\infty$ e apresentamos critérios necessários e suficientes para a in/finitude quase-certa do número de espécies presentes em tal distribuição. Na Seção 4.1, construímos uma escada de recordes, no sentido inverso do tempo, usando o processo de extinções e limiares associados. Na Seção 4.2, demonstramos o Teorema 2, enunciado na Seção 2.2. Na Seção 4.3, calculamos a transformada de Laplace do número de surgimentos acima da escada de limiares recordes e apresentamos critérios equivalentes para a in/finitude quase-certa da mesma variável aleatória . Na Seção 4.4, demonstramos o Teorema 3, enunciado na Seção 2.2. Por fim, na Seção 4.5, revisitamos o exemplo com distribuições exponenciais, apresentado na Seção 3.5.

\subsection{Escada de limiares recordes}

Nesta seção, usamos o processo de extinções e os limiares associados para construir uma escada de recordes. Considere os índices recordes $\left(J_{k}(0)\right)_{k \geq 1}$ definidos em (2.1):

$$
\begin{aligned}
J_{1}(0) & =\max \left\{j: S_{j} \leq 0\right\}=-1 \\
J_{k+1}(0) & =\max \left\{j<J_{k}(0): Y_{j}>Y_{J_{k}}(0)\right\} \quad \text { para cada } \quad k \geq 1
\end{aligned}
$$

Proposição 7. Os pontos $\left(Y_{J_{k}}(0)\right)_{k \geq 1}$ formam um processo de Poisson em $\mathbb{R}^{+}$com intensidade $\int_{B} R_{\dagger}(d x)$, onde $B \in \mathcal{B}\left(\mathbb{R}^{+}\right)$.

Este resultado é análogo à Proposição 1, cuja demonstração pode ser encontrada em Resnick (1973). Veja também a Observação 5, no apêndice.

Denotamos por $S_{J_{k}}(0)$ o instante do $k$-ésimo recorde e por $\Delta S_{J_{k}}(0) \doteq S_{J_{k}}(0)-S_{J_{k+1}}(0)$ o intervalo entre dois recordes consecutivos.

Proposição 8. Os pontos $\left(Y_{J_{k}}(0), \Delta S_{J_{k}}(0)\right)_{k \geq 1}$ formam um processo de Poisson em $[0,+\infty)^{2}$ com intensidade:

$$
\hat{\mu}_{\dagger}(C) \doteq \iint_{C} \lambda_{\dagger} \overline{F_{\dagger}}(x) e^{-\lambda_{\dagger} \overline{F_{\dagger}}(x) s} d s R_{\dagger}(d x) \quad \text { para cada } \quad C \in \mathcal{B}\left([0,+\infty)^{2}\right)
$$

Demonstração. Este resultado é análogo à Proposição 2 e segue do Teorema das Marcas, enunciado na Seção A.2, com marcação dada pelo kernel $K_{\dagger}$ definido da mesma forma que $K_{\star}$, mas com $\left(\lambda_{\dagger}, \overline{F_{\dagger}}\right)$ no lugar de $\left(\lambda_{\star}, \overline{F_{\star}}\right)$. A definição de kernel pode ser encontrada na Seção A.2.

Defina as regiões aleatórias acima de cada degrau da escada por:

$$
\begin{aligned}
& E_{0} \doteq\left[S_{J_{1}}(0), 0\right) \times[0,+\infty) \\
& E_{k} \doteq\left[S_{J_{k+1}}(0), S_{J_{k}}(0)\right) \times\left[Y_{J_{k}}(0),+\infty\right) \quad \text { para } \quad k \geq 1
\end{aligned}
$$


A Figura 4.1 mostra uma possível realização dos processos, com a escada de limiares recordes e alguns eventos de surgimento.

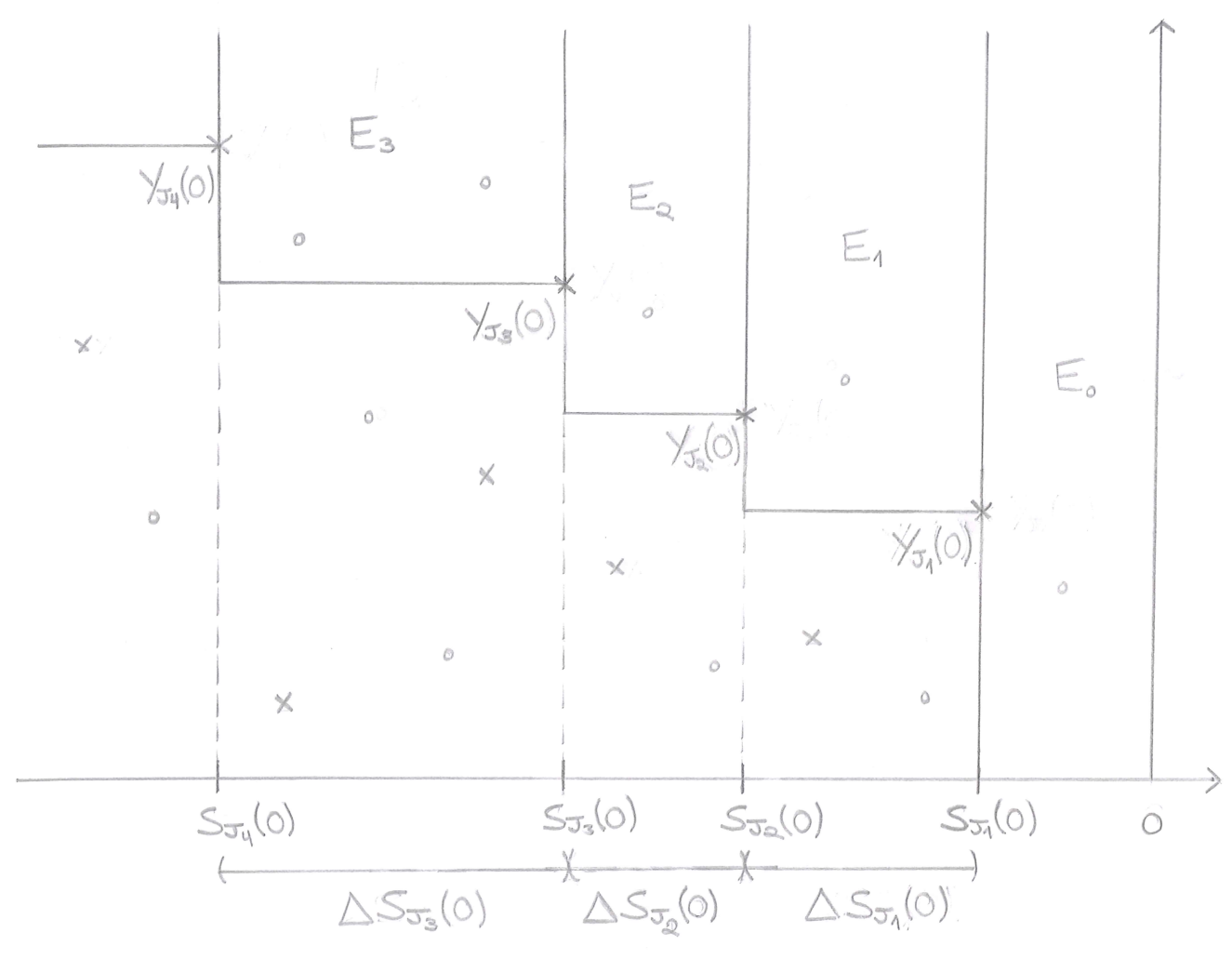

Figura 4.1: Possível realização da escada de limiares recordes com alguns eventos de surgimento

Proposição 9. Os pontos $\left(T_{-i}, X_{-i}\right)_{i \geq 1}$ formam um processo de Poisson em $(-\infty, 0] \times[0,+\infty)$ denotado por $\hat{\Pi}_{\star}$ com intensidade:

$$
\mu_{\star}\left(C^{\prime}\right) \doteq \iint_{C^{\prime}} \lambda_{\star} d t F_{\star}(d y) \quad \text { para cada } \quad C^{\prime} \in \mathcal{B}((-\infty, 0] \times[0,+\infty))
$$

Demonstração. Este resultado é análogo à Proposição 3 e segue do Teorema das Marcas, enunciado na Seção A.2, com $\mathbb{Q}_{\star}$-marcação independente, onde $\mathbb{Q}_{\star}$ é a medida induzida por $X$. As definições podem ser encontradas na Seção A.2.

Defina as variáveis aleatórias $\left(\Sigma_{k}\right)_{k \geq 0}$ e $\left(N_{k}\right)_{k \geq 0}$ por:

$$
\begin{aligned}
& \Sigma_{k} \doteq \mu_{\star}\left(E_{k}\right) \\
& \text { para cada } k \geq 0 \\
& N_{k} \doteq \#\left\{E_{k} \cap \hat{\Pi}_{\star}\right\} \\
& \text { para cada } k \geq 0
\end{aligned}
$$

Defina a região aleatória $E$ e as variáveis aleatórias $\Sigma$ e $N$ por:

$$
\begin{aligned}
& E \doteq \bigcup_{k \geq 1} E_{k} \\
& \Sigma \doteq \mu_{\star}(E) \\
& N \doteq \#\left\{E \cap \hat{\Pi}_{\star}\right\}
\end{aligned}
$$

Observe que $N$ é o número de eventos de surgimento acima da escada de limiares recordes. 
Note que a escada de limiares recordes $E$ tem estrutura idêntica à escada de aptidões recordes $D$, apesar de estar no segundo quadrante. Além disso, as variáveis aleatórias $\Sigma$ e $N$ são definidas da mesma forma que $\Lambda$ e $M$. Assim, valem os mesmos resultados nos dois casos, sempre invertendo as posições de $\left(\lambda_{\star}, \overline{F_{\star}}\right)$ e $\left(\lambda_{\dagger}, \overline{F_{\dagger}}\right)$, como mencionado na Observação 1.

\subsection{Demonstração do Teorema 2}

Nesta seção, apresentamos a demonstração do Teorema 2, enunciado na Seção 2.2. A demonstração segue o seguinte raciocínio: observando que $\eta_{-t}(0)$ e $\eta_{0}(t)$ têm a mesma distribuição, é suficiente considerar a convergência do primeiro; para mostrar a convergência forte deste processo, basta separá-lo em duas componentes, uma com condição inicial vazia e outra contendo somente as espécies da configuração inicial; as espécies presentes na condição inicial serão eliminadas do sistema em tempo quase-certamente finito, enquanto a componente com condição inicial vazia converge fortemente para a distribuição limite.

Demonstração. A distribuição do processo $\eta_{t}(t+s)$ depende somente de $s>0$, já que os processos $\Pi_{\star}$ e $\Pi_{\dagger}$ são homogêneos e as variáveis aleatórias $X_{i}$ e $Y_{j}$ são independentes dos instantes $T_{i}$ e $S_{j}$.

Logo, a família de processos $\eta_{t}=\left\{\eta_{t}(t+s): s \geq 0\right\}$ tem a mesma distribuição para cada $t \in \mathbb{R}$. Em particular:

$$
\mathbb{P}\left(\eta_{0}(t) \in \bar{A} \mid \eta_{0}(0)=A\right)=\mathbb{P}\left(\eta_{-t}(0) \in \bar{A} \mid \eta_{-t}(-t)=A\right)
$$

onde A é um subconjunto localmente finito de $[0,+\infty)$ e $\bar{A}$ é um elemento da sigma-álgebra apropriada. Para mais informações sobre processos pontuais, consulte Last e Penrose (2017).

Sejam $\left(t_{l}\right)_{l \geq 1}$ uma sequência crescente em $\mathbb{R}^{+}$tal que $t_{l} \rightarrow+\infty$ quando $l \rightarrow+\infty$ e $A$ um subconjunto localmente finito de $\mathbb{R}^{+}$. Considere a sequência de processos $\left(\eta_{-t_{l}}\right)_{l \geq 1}$ com condição inicial:

$$
\eta_{-t_{l}}\left(-t_{l}\right)=A \quad \text { para cada } \quad l \geq 1
$$

Vamos definir um processo auxiliar, denotado por $\eta^{\prime}$, com a mesma estrutura do processo original, mas com condição inicial vazia. Defina a sequência $\left(\eta_{l}^{\prime}\right)_{l \geq 1}$ por:

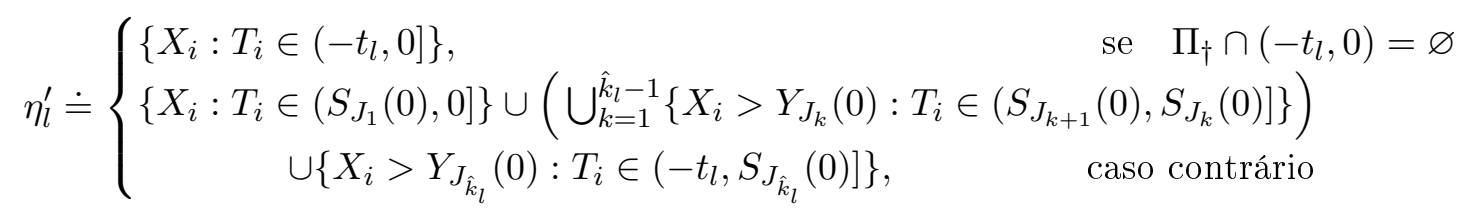

onde $\hat{k}_{l} \doteq \max \left\{k: S_{J_{k}}(0)>-t_{l}\right\}$ e $\bigcup_{k=1}^{\hat{k}_{l}-1}\left\{X_{i}>Y_{J_{k}}(0): T_{i} \in\left(S_{J_{k+1}}(0), S_{J_{k}}(0)\right]\right\}=\varnothing$ se $\hat{k}_{l}=1$.

Note que $\left(\hat{k}_{l}\right)_{l \geq 1}$ é crescente com $\hat{k}_{l} \rightarrow+\infty$ quando $l \rightarrow+\infty$. Assim, a sequência $\left(\eta_{l}^{\prime}\right)_{l \geq 1}$ é crescente e vale:

$$
\lim _{l \rightarrow+\infty} \eta_{l}^{\prime}=\bigcup_{l \geq 1} \eta_{l}^{\prime}=\hat{\eta}
$$

Onde $\hat{\eta}$ é definido por:

$$
\hat{\eta} \doteq\left\{X_{i}: T_{i} \in\left(S_{J_{1}}(0), 0\right]\right\} \cup\left(\bigcup_{k \geq 1}\left\{X_{i}>Y_{J_{k}}(0): T_{i} \in\left(S_{J_{k+1}}(0), S_{J_{k}}(0)\right]\right\}\right)
$$

Defina a sequência $\left(\eta_{l}^{\prime \prime}\right)_{l \geq 1}$ por:

$$
\eta_{l}^{\prime \prime} \doteq \begin{cases}A, & \text { se } \Pi_{\dagger} \cap\left(-t_{l}, 0\right)=\varnothing \\ A \cap\left(Y_{J_{\hat{k}_{l}}}(0),+\infty\right), & \text { caso contrário }\end{cases}
$$

Segue da Proposição 7 que $Y_{J_{k}}(0) \rightarrow+\infty$ quando $k \rightarrow+\infty$, já que $R_{\dagger}(x) \rightarrow+\infty$ quando

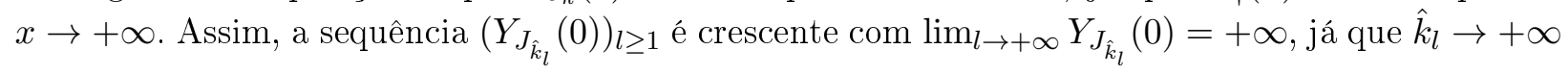


quando $l \rightarrow+\infty$. Logo, a sequência $\left(\eta_{l}^{\prime \prime}\right)_{l \geq 1}$ é decrescente e vale:

$$
\lim _{l \rightarrow+\infty} \eta_{l}^{\prime \prime}=\bigcap_{l \geq 1} \eta_{l}^{\prime \prime}=\varnothing
$$

Como:

$$
\eta_{-t_{l}}(0)=\eta_{l}^{\prime} \cup \eta_{l}^{\prime \prime} \quad \text { para cada } \quad l \geq 1
$$

Segue que:

$$
\lim _{l \rightarrow+\infty} \eta_{-t_{l}}(0)=\lim _{l \rightarrow+\infty} \eta_{l}^{\prime} \cup \lim _{l \rightarrow+\infty} \eta_{l}^{\prime \prime}=\hat{\eta}
$$

Como o limite não depende da escolha de $\left(t_{l}\right)_{l \geq 1}$, temos que:

$$
\lim _{t \rightarrow+\infty} \eta_{-t}(0)=\hat{\eta} \quad \text { quase certamente }
$$

Valendo a convergência quase certa, também vale:

$$
\eta_{-t}(0) \rightarrow \hat{\eta} \quad \text { em distribuição, quando } \quad t \rightarrow+\infty
$$

Por (4.10), temos que:

$$
\eta_{0}(t) \rightarrow \hat{\eta} \quad \text { em distribuição, quando } \quad t \rightarrow+\infty
$$

\subsection{O número de espécies presentes na distribuição limite}

Nesta seção, calculamos a transformada de Laplace do número de surgimentos acima da escada de limiares recordes, via funcional de Laplace, e apresentamos critérios equivalentes para a in/finitude quase-certa da mesma variável aleatória. Note que isto é um estudo preliminar do número de espécies presentes na distribuição limite $\hat{\eta}$, pois segue da definição em (4.14) que:

$$
\# \hat{\eta}=\sum_{k \geq 0} \#\left\{E_{k} \cap \hat{\Pi}_{\star}\right\}=\sum_{k \geq 0} N_{k}=N_{0}+N
$$

Assim, para avaliar a in/finitude de $\# \hat{\eta}$, devemos primeiro avaliar a in/finitude de $N$.

Note que $N$ tem distribuição de Poisson com parâmetro $\Sigma$. Logo, $N$ tem distribuição misturada.

Proposição 10. A tranformada de Laplace de $N$ pode ser escrita na forma:

$$
\mathbb{E}\left[e^{-t N}\right]=\mathbb{E}\left[e^{-\left(1-e^{-t}\right) \Sigma}\right]
$$

Demonstração. Este resultado é análogo à Proposição 4.

Defina a função $h_{\star}:[0,+\infty)^{2} \rightarrow \mathbb{R}^{+}$por:

$$
h_{\star}(x, s) \doteq \lambda_{\star} s \overline{F_{\star}}(x)
$$

A variável aleatória $\Sigma$ pode ser escrita como uma soma:

$$
\Sigma=\mu_{\star}(E)=\sum_{k \geq 1} \mu_{\star}\left(E_{k}\right)=\sum_{k \geq 1} \lambda_{\star} \Delta S_{J_{k}}(0) \overline{F_{\star}}\left(Y_{J_{k}}(0)\right)=\sum_{k \geq 1} h_{\star}\left(Y_{J_{k}}(0), \Delta S_{J_{k}}(0)\right)
$$

Proposição 11. A transformada de Laplace de $N$ é:

$$
\mathbb{E}\left[e^{-t N}\right]=\exp \left[-\int_{[0,+\infty)} \frac{\left(1-e^{-t}\right) \lambda_{\star} \overline{F_{\star}}(x)}{\lambda_{\dagger} \overline{F_{\dagger}}(x)+\left(1-e^{-t}\right) \lambda_{\star} \overline{F_{\star}}(x)} R_{\dagger}(d x)\right]
$$


Demonstração. Este resultado é análogo à Proposição 5 e segue da caracterização de processos de Poisson por funcional de Laplace, enunciada na Seção A.2, com as posições de $\left(\lambda_{\star}, \overline{F_{\star}}\right)$ e $\left(\lambda_{\dagger}, \overline{F_{\dagger}}\right)$ invertidas.

Defina as funções $\bar{\phi}: \mathbb{R}^{+} \rightarrow \mathbb{R}^{+}$e $\bar{\psi}:[0,1] \rightarrow[0,1]$ por:

$$
\begin{aligned}
\bar{\phi}(t) & \doteq \int_{[0,+\infty)} \frac{\left(1-e^{-t}\right) \lambda_{\star} \overline{F_{\star}}(x)}{\lambda_{\dagger} \overline{F_{\dagger}}(x)+\left(1-e^{-t}\right) \lambda_{\star} \overline{F_{\star}}(x)} R_{\dagger}(d x) \\
\bar{\psi}(u) & \doteq \overline{F_{\star}} \circ{\overline{F_{\dagger}}}^{-1}(u)
\end{aligned}
$$

Denote por $\bar{\phi}(+\infty) \in[0,+\infty]$ o seguinte limite:

$$
\bar{\phi}(+\infty) \doteq \lim _{t \rightarrow+\infty} \bar{\phi}(t)
$$

Pelo Teorema de Convergência Monótona:

$$
\bar{\phi}(+\infty)=\int_{[0,+\infty)} \frac{\lambda_{\star} \overline{F_{\star}}(x)}{\lambda_{\dagger} \overline{F_{\dagger}}(x)+\lambda_{\star} \overline{F_{\star}}(x)} R_{\dagger}(d x)
$$

Podemos calcular $\mathbb{E}[N]$ usando a Fórmula de Campbell, enunciada na Seção A.2, resolvendo uma integral análoga à $(3.25)$ com as posições de $\left(\lambda_{\star}, \overline{F_{\star}}\right)$ e $\left(\lambda_{\dagger}, \overline{F_{\dagger}}\right)$ invertidas:

$$
\mathbb{E}[N]=\frac{\lambda_{\star}}{\lambda_{\dagger}} \int_{[0,+\infty)} \frac{\overline{F_{\star}}(x)}{\overline{F_{\dagger}}(x)} R_{\dagger}(d x)
$$

Proposição 12. São equivalentes:

i) $\bar{\phi}(+\infty)<+\infty$

ii) $\mathbb{P}(N<+\infty)=1$

iii) $\mathbb{E}[N]<+\infty$

iv) $\frac{\bar{\psi}(u)}{u^{2}}$ é integrável na origem

v) $\bar{\psi}\left(s^{-1}\right)$ é integrável no infinito

Demonstração. Este resultado é análogo à Proposição 6 e pode ser demonstrado seguindo o mesmo raciocínio, trocando os papéis de $\left(\lambda_{\star}, \overline{F_{\star}}\right)$ e $\left(\lambda_{\dagger}, \overline{F_{\dagger}}\right)$.

\subsection{Demonstração do Teorema 3}

Nesta seção, apresentamos a demonstração do Teorema 3, enunciado na Seção 2.2. Observe que a Proposição 12 fornece critérios necessários e suficientes para a in/finitude quase-certa do número de espécies na região acima da escada, excluindo o degrau inicial. Para avaliar a in/finitude do número de espécies presentes na distribuição limite, basta incluir as espécies neste degrau, em quantidade quase-certamente finita, quaisquer que sejam as distribuições das aptidões e limiares.

Demonstração. Note que $N_{0}$ tem distribuição de Poisson com parâmetro $\Sigma_{0}$ :

$$
\mathbb{P}\left(N_{0}=n \mid \Sigma_{0}=\mu\right)=\frac{\mu^{n}}{n !} e^{-\mu}
$$

Daí seguem:

$$
\begin{aligned}
\mathbb{E}\left[N_{0} \mid \Sigma_{0}=\mu\right] & =\mu \\
\mathbb{E}\left[N_{0} \mid \Sigma_{0}\right] & =\Sigma_{0}
\end{aligned}
$$


Logo:

$$
\mathbb{E}\left[N_{0}\right]=\mathbb{E}\left[\mathbb{E}\left[N_{0} \mid \Sigma_{0}\right]\right]=\mathbb{E}\left[\Sigma_{0}\right]=\frac{\lambda_{\star}}{\lambda_{\dagger}}<+\infty
$$

Já que:

$$
\Sigma_{0}=\mu_{\star}\left(E_{0}\right)=\lambda_{\star}\left(0-S_{-1}\right) \sim \operatorname{Exponencial}\left(\frac{\lambda_{\dagger}}{\lambda_{\star}}\right)
$$

Assim:

$$
\mathbb{P}\left(N_{0}<+\infty\right)=1
$$

Como vimos em (4.22), temos:

$$
\# \hat{\eta}=N_{0}+N
$$

Pela Proposiç̧̃ão 12, se $\int_{[0,+\infty)} \frac{\overline{F_{\star}}(x)}{\overline{F_{\dagger}}(x)} R_{\dagger}(d x)<+\infty$, então $\mathbb{P}(N<+\infty)=1$. Logo:

$$
\# \hat{\eta}<+\infty \quad \text { quase certamente }
$$

Pela Proposição 12, se $\int_{[0,+\infty)} \frac{\overline{F_{\star}}(x)}{\overline{F_{\dagger}}(x)} R_{\dagger}(d x)=+\infty$, então $\mathbb{P}(N=+\infty)=1$. Logo:

$$
\# \hat{\eta}=+\infty \quad \text { quase certamente }
$$

\subsection{Exemplo}

Nesta seção, vamos revisitar o exemplo da Seção 3.5 e calcular a distribuição de \# $\hat{\eta}$.

Suponha que:

$$
\begin{aligned}
& \overline{F_{\star}}(x)=e^{-\alpha_{\star} x} \mathbf{1}_{[0,+\infty)}(x) \\
& \overline{F_{\dagger}}(x)=e^{-\alpha_{\dagger} x} \mathbf{1}_{[0,+\infty)}(x)
\end{aligned}
$$

Note que:

$$
\int_{0}^{+\infty} \frac{\overline{F_{\star}}(x)}{\overline{F_{\dagger}}(x)} R_{\dagger}(d x)=\left\{\begin{array}{lll}
\frac{\alpha_{\dagger}}{\alpha_{\star}-\alpha_{\dagger}}, & \text { se } & \alpha_{\star}>\alpha_{\dagger} \\
+\infty, & \text { se } & \alpha_{\star} \leq \alpha_{\dagger}
\end{array}\right.
$$

Para $\alpha_{\star} \leq \alpha_{\dagger}$, temos $\# \hat{\eta}=+\infty$ quase certamente, pelo Teorema 3 .

Para $\alpha_{\star}>\alpha_{\dagger}$, o mesmo raciocínio que levou a (3.76), pode ser usado para mostrar que $N$ tem distribuição binomial negativa com parâmetros $\bar{r}$ e $q$, onde $\bar{r}=\frac{\alpha_{\dagger}}{\alpha_{\star}-\alpha_{\dagger}}$ e $q=\frac{\lambda_{\star}}{\lambda_{\star}+\lambda_{\dagger}}=1-p$.

Segue de (4.32) e (4.36) que:

$$
\begin{gathered}
\mathbb{P}\left(N_{0}=n\right)=\mathbb{E}\left[\mathbb{P}\left(N_{0}=n \mid \Sigma_{0}\right)\right]=\mathbb{E}\left[\frac{\Sigma_{0}^{n}}{n !} e^{-\Sigma_{0}}\right]=\int_{0}^{+\infty} \frac{\mu^{n}}{n !} e^{-\mu} \frac{\lambda_{\dagger}}{\lambda_{\star}} e^{-\frac{\lambda_{\dagger}}{\lambda_{\star}} \mu} d \mu \\
=\left(\frac{\lambda_{\star}}{\lambda_{\star}+\lambda_{\dagger}}\right)^{n} \frac{\lambda_{\dagger}}{\lambda_{\star}+\lambda_{\dagger}}=q^{n}(1-q)
\end{gathered}
$$

Logo, $N_{0}$ tem distribuição binomial negativa com parâmetros 1 e $q$.

Vamos mostrar que $N_{0}$ e $N$ são independentes. Note que:

$$
\mathbb{P}\left(N_{0}=n_{0}, N=n \mid \Sigma_{0}=\mu_{0}, \Sigma=\mu\right)=\mathbb{P}\left(N_{0}=n_{0} \mid \Sigma_{0}=\mu_{0}\right) \mathbb{P}(N=n \mid \Sigma=\mu)
$$

Além disso, $\Sigma_{0}=-\lambda_{\star} S_{J_{1}}(0)$ e $\Sigma=\sum_{k \geq 1} h_{\star}\left(Y_{J_{k}}(0), \Delta S_{J_{k}}(0)\right)$ são independentes, já que a sequência $\left(Y_{J_{k}}(0), \Delta S_{J_{k}}(0)\right)_{k \geq 1}$ é independente de $S_{J_{1}}(0)=S_{-1}$. Daí segue que as sigma-álgebras 
$\sigma\left(\Sigma_{0}\right)$ e $\sigma(\Sigma)$ são independentes.

Como $\mathbb{P}\left(N_{0}=n_{0} \mid \Sigma_{0}\right)$ é $\sigma\left(\Sigma_{0}\right)$-mensurável e $\mathbb{P}(N=n \mid \Sigma)$ é $\sigma(\Sigma)$-mensurável, temos que:

$$
\begin{aligned}
\mathbb{P}\left(N_{0}=n_{0}, N=n\right) & =\mathbb{E}\left[\mathbb{P}\left(N_{0}=n_{0}, N=n \mid \Sigma_{0}, \Sigma\right)\right]=\mathbb{E}\left[\mathbb{P}\left(N_{0}=n_{0} \mid \Sigma_{0}\right) \mathbb{P}(N=n \mid \Sigma)\right] \\
& =\mathbb{E}\left[\mathbb{P}\left(N_{0}=n_{0} \mid \Sigma_{0}\right)\right] \mathbb{E}[\mathbb{P}(N=n \mid \Sigma)]=\mathbb{P}\left(N_{0}=n_{0}\right) \mathbb{P}(N=n)
\end{aligned}
$$

Logo, $N_{0}$ e $N$ são independentes. Segue de (4.22) que \# $\hat{\eta}$ tem distribuição binomial negativa com parâmetros $1+\bar{r}=\frac{\alpha_{\star}}{\alpha_{\star}-\alpha_{\dagger}}$ e $q=\frac{\lambda_{\star}}{\lambda_{\star}+\lambda_{\dagger}}$.

Observação 4. Invertendo os papéis de $\left(\lambda_{\star}, \overline{F_{\star}}\right)$ e $\left(\lambda_{\dagger}, \overline{F_{\dagger}}\right)$ na Observação 2, vemos que:

$$
\bar{\phi}(t)=\int_{0}^{1} \frac{\left(1-e^{-t}\right) \lambda_{\star} \bar{\psi}(u)}{\lambda_{\dagger} u+\left(1-e^{-t}\right) \lambda_{\star} \bar{\psi}(u)} \frac{d u}{u}
$$

Assim, a distribuição de $N$ depende somente de $\bar{\psi}$. Pelo mesmo argumento da Observação $3, N$ tem distribuição binomial negativa com parâmetros $\bar{r}$ e $q$ sempre que:

$$
\overline{F_{\star}}(x)=\overline{F_{\dagger}}(x)^{\frac{\alpha_{\star}}{\alpha_{\dagger}}}
$$

Como a distribuição de $N_{0}$ não depende das distribuições $F_{\star}$ e $F_{\dagger}$, a condição acima garante que $\# \hat{\eta}$ tem distribuição binomial negativa com parâmetros $1+\bar{r}$ e $q$. 


\section{Apêndice A}

\section{Complementos}

\section{A.1 Recordes como processo de Poisson}

Nesta seção, apresentamos uma demonstração da Proposição 1, enunciada na Seção 3.1.

Demonstração. Defina a sequência de variáveis aleatórias $\left(\xi_{i}\right)_{i \geq 1}$ por:

$$
\xi_{i} \doteq R_{\star}\left(X_{i}\right)=-\log \overline{F_{\star}}\left(X_{i}\right) \quad \text { para cada } i \geq 1
$$

Como a sequência $\left(X_{i}\right)_{i \geq 1}$ é i.i.d., a sequência $\left(\xi_{i}\right)_{i \geq 1}$ também é i.i.d. Além disso, as variáveis aleatórias $\xi_{i}$ têm distribuição exponencial de parâmetro 1, para cada $i \geq 1$, já que:

$$
\begin{aligned}
\mathbb{P}\left(\xi_{1} \leq x\right) & =\mathbb{P}\left(-\log \overline{F_{\star}}\left(X_{1}\right) \leq x\right)=\mathbb{P}\left(\overline{F_{\star}}\left(X_{1}\right) \geq e^{-x}\right)=\mathbb{P}\left(F_{\star}\left(X_{1}\right) \leq 1-e^{-x}\right) \\
& =\mathbb{P}\left(X_{1} \leq F_{\star}^{-1}\left(1-e^{-x}\right)\right)=F_{\star}\left(F_{\star}^{-1}\left(1-e^{-x}\right)\right)=1-e^{-x}, \quad \text { para } \quad x>0
\end{aligned}
$$

Como $R_{\star}$ é uma função crescente, os recordes da sequência $\left(\xi_{i}\right)_{i \geq 1}$ ocorrem nos mesmos índices que os recordes da sequência $\left(X_{i}\right)_{i \geq 1}$. Assim, $\left(\xi_{I_{k}}\right)_{k \geq 1}$ é a sequência dos recordes de $\left(\xi_{i}\right)_{i \geq 1}$.

Seja $f_{n}\left(x_{1}, \ldots, x_{n}\right)$ a densidade do vetor $\left(\xi_{I_{1}}, \ldots, \xi_{I_{n}}\right)$ para cada $n \geq 1$. Vamos mostrar que:

$$
f_{n}\left(x_{1}, \ldots, x_{n}\right)=e^{-x_{n}} \mathbf{1}_{\left\{0<x_{1}<\ldots<x_{n}\right\}} \quad \text { para cada } n \geq 1
$$

Como $\xi_{I_{1}}=\xi_{1}$ é exponencial de parâmetro 1, vale:

$$
f_{1}\left(x_{1}\right)=e^{-x_{1}} \mathbf{1}_{\left\{x_{1}>0\right\}}
$$

Suponha que vale (A.3) para algum $n \geq 1$. Vamos mostrar que também vale para $n+1$.

Para $0<x_{n}<x_{n+1}$, vale:

$$
\begin{aligned}
\mathbb{P}\left(\xi_{I_{n+1}} \leq x_{n+1} \mid \xi_{I_{n}}=x_{n}\right) & =\sum_{j \geq 1} \mathbb{P}\left(\xi_{I_{n+1}} \leq x_{n+1}, I_{n+1}=I_{n}+j \mid \xi_{I_{n}}=x_{n}\right) \\
& =\sum_{j \geq 1} \mathbb{P}\left(\xi_{I_{n}+1} \leq x_{n}, \ldots, \xi_{I_{n}+j-1} \leq x_{n}, x_{n}<\xi_{I_{n}+j} \leq x_{n+1} \mid \xi_{I_{n}}=x_{n}\right) \\
& =\sum_{j \geq 1} \mathbb{P}\left(\xi_{2} \leq x_{n}, \ldots, \xi_{j} \leq x_{n}, x_{n}<\xi_{j+1} \leq x_{n+1} \mid \xi_{1}=x_{n}\right) \\
& =\sum_{j \geq 1} \mathbb{P}\left(\xi_{2} \leq x_{n}, \ldots, \xi_{j} \leq x_{n}, x_{n}<\xi_{j+1} \leq x_{n+1}\right) \\
& =\sum_{j \geq 1} \mathbb{P}\left(\xi_{2} \leq x_{n}\right) \ldots \mathbb{P}\left(\xi_{j} \leq x_{n}\right) \mathbb{P}\left(x_{n}<\xi_{j+1} \leq x_{n+1}\right) \\
& =\left(1-e^{-x_{n+1}}-1+e^{-x_{n}}\right) \sum_{j \geq 1}\left(1-e^{-x_{n}}\right)^{j-1}
\end{aligned}
$$




$$
=\left(e^{-x_{n}}-e^{-x_{n+1}}\right) e^{x_{n}}=1-e^{-\left(x_{n+1}-x_{n}\right)}
$$

onde as passagens podem ser justificadas por particionamento do evento $\left\{\xi_{I_{n+1}} \leq x_{n+1}\right\}$ pelo número de tentativas até que se observe o novo recorde e por distribuição idêntica da sequência original.

Como $0<\xi_{I_{n}}<\xi_{I_{n+1}}$ quase certamente, temos:

$$
\mathbb{P}\left(\xi_{I_{n+1}} \leq x_{n+1} \mid \xi_{I_{n}}=x_{n}\right)=\left(1-e^{-\left(x_{n+1}-x_{n}\right)}\right) \mathbf{1}_{\left\{0<x_{n}<x_{n+1}\right\}}
$$

Note que:

$$
\mathbb{P}\left(\xi_{I_{n+1}} \leq x_{n+1} \mid \xi_{I_{1}}=x_{1}, \ldots, \xi_{I_{n}}=x_{n}\right)=\mathbb{P}\left(\xi_{I_{n+1}} \leq x_{n+1} \mid \xi_{I_{n}}=x_{n}\right)
$$

Daí segue que:

$$
\begin{aligned}
f_{n+1 \mid n}\left(x_{n+1} \mid \xi_{I_{1}}=x_{1}, \ldots, \xi_{I_{n}}=x_{n}\right) & =\frac{d}{d x_{n+1}} \mathbb{P}\left(\xi_{I_{n+1}} \leq x_{n+1} \mid \xi_{I_{n}}=x_{n}\right) \\
& =\frac{d}{d x_{n+1}}\left(1-e^{-\left(x_{n+1}-x_{n}\right)}\right) \mathbf{1}_{\left\{0<x_{n}<x_{n+1}\right\}} \\
& =e^{-\left(x_{n+1}-x_{n}\right)} \mathbf{1}_{\left\{0<x_{n}<x_{n+1}\right\}}
\end{aligned}
$$

Logo:

$$
\begin{aligned}
f_{n+1}\left(x_{1}, \ldots, x_{n+1}\right) & =f_{n+1 \mid n}\left(x_{n+1} \mid \xi_{I_{1}}=x_{1}, \ldots, \xi_{I_{n}}=x_{n}\right) f_{n}\left(x_{1}, \ldots, x_{n}\right) \\
& =e^{-\left(x_{n+1}-x_{n}\right)} \mathbf{1}_{\left\{0<x_{n}<x_{n+1}\right\}} e^{-x_{n}} \mathbf{1}_{\left\{0<x_{1}<\ldots<x_{n}\right\}} \\
& =e^{-x_{n+1}} \mathbf{1}_{\left\{0<x_{1}<\ldots<x_{n+1}\right\}}
\end{aligned}
$$

Assim, (A.3) vale para $n+1$. Por indução, (A.3) vale para cada $n \geq 1$.

Seja $\left(\zeta_{i}\right)_{i \geq 1}$ uma sequência i.i.d. de variáveis aleatórias exponenciais de parâmetro 1 .

Defina a sequência $\left(Z_{k}\right)_{k \geq 1}$ por:

$$
Z_{k} \doteq \sum_{i=1}^{k} \zeta_{i} \quad \text { para cada } \quad k \geq 1
$$

Seja $g_{n}\left(x_{1}, \ldots, x_{n}\right)$ a densidade do vetor $\left(Z_{1}, \ldots, Z_{n}\right)$ para cada $n \geq 1$. Vamos mostrar que:

$$
g_{n}\left(x_{1}, \ldots, x_{n}\right)=e^{-x_{n}} \mathbf{1}_{\left\{0<x_{1}<\ldots<x_{n}\right\}} \quad \text { para cada } n \geq 1
$$

Como $Z_{1}=\zeta_{1}$ é exponencial de parâmetro 1 , vale:

$$
g_{1}\left(x_{1}\right)=e^{-x_{1}} \mathbf{1}_{\left\{x_{1}>0\right\}}
$$

Suponha que vale (A.10) para algum $n \geq 1$. Vamos mostrar que também vale para $n+1$.

Como $0<Z_{n}<Z_{n+1}$ quase certamente, temos:

$$
\mathbb{P}\left(Z_{n+1} \leq x_{n+1} \mid Z_{n}=x_{n}\right)=\mathbb{P}\left(\zeta_{n+1} \leq x_{n+1}-x_{n}\right)=\left(1-e^{-\left(x_{n+1}-x_{n}\right)}\right) \mathbf{1}_{\left\{0<x_{n}<x_{n+1}\right\}}
$$

Note que:

$$
\mathbb{P}\left(Z_{n+1} \leq x_{n+1} \mid Z_{1}=x_{1}, \ldots, Z_{n}=x_{n}\right)=\mathbb{P}\left(Z_{n+1} \leq x_{n+1} \mid Z_{n}=x_{n}\right)
$$


Daí segue que:

$$
\begin{aligned}
g_{n+1 \mid n}\left(x_{n+1} \mid Z_{1}=x_{1}, \ldots, Z_{n}=x_{n}\right) & =\frac{d}{d x_{n+1}} \mathbb{P}\left(Z_{n+1} \leq x_{n+1} \mid Z_{n}=x_{n}\right) \\
& =\frac{d}{d x_{n+1}}\left(1-e^{-\left(x_{n+1}-x_{n}\right)}\right) \mathbf{1}_{\left\{0<x_{n}<x_{n+1}\right\}} \\
& =e^{-\left(x_{n+1}-x_{n}\right)} \mathbf{1}_{\left\{0<x_{n}<x_{n+1}\right\}}
\end{aligned}
$$

Logo:

$$
\begin{aligned}
g_{n+1}\left(x_{1}, \ldots, x_{n+1}\right) & =g_{n+1 \mid n}\left(x_{n+1} \mid Z_{1}=x_{1}, \ldots, Z_{n}=x_{n}\right) g_{n}\left(x_{1}, \ldots, x_{n}\right) \\
& =e^{-\left(x_{n+1}-x_{n}\right)} \mathbf{1}_{\left\{0<x_{n}<x_{n+1}\right\}} e^{-x_{n}} \mathbf{1}_{\left\{0<x_{1}<\ldots<x_{n}\right\}} \\
& =e^{-x_{n+1}} \mathbf{1}_{\left\{0<x_{1}<\ldots<x_{n+1}\right\}}
\end{aligned}
$$

Assim, (A.10) vale para $n+1$. Por indução, (A.10) vale para cada $n \geq 1$. Note que:

$$
f_{n}\left(x_{1}, \ldots, x_{n}\right)=g_{n}\left(x_{1}, \ldots, x_{n}\right) \quad \text { para cada } n \geq 1
$$

Daí segue que as sequências $\left(\xi_{I_{k}}\right)_{k \geq 1}$ e $\left(Z_{k}\right)_{k \geq 1}$ são identicamente distribuidas.

Assim, a sequência $\left(\xi_{I_{k}}\right)_{k \geq 1}$ forma um processo de Poisson homogêneo de taxa $1 \mathrm{em} \mathbb{R}^{+}$, já que o mesmo ocorre com a sequência $\left(Z_{k}\right)_{k \geq 1}$.

Denote a função inversa de $R_{\star}$ por $Q_{\star}: \mathbb{R}^{+} \rightarrow \mathbb{R}^{+}$e note que:

$$
Q_{\star}\left(\xi_{I_{k}}\right)=X_{I_{k}} \quad \text { para cada } \quad k \geq 1
$$

Pelo Teorema da Transformação, que pode ser encontrado em Last e Penrose (2017), a sequência $\left(X_{I_{k}}\right)$ forma um processo de Poisson em $\mathbb{R}^{+}$com intensidade:

$$
\begin{aligned}
& \left|Q_{\star}^{-1}(a, b]\right|=\left|\left(R_{\star}(a), R_{\star}(b)\right]\right|=R_{\star}(b)-R_{\star}(a) \quad \text { para cada } \quad(a, b] \subset \mathbb{R}^{+} \\
& \left|Q_{\star}^{-1}(B)\right|=\int_{B} R_{\star}(d x) \quad \text { para cada } B \in \mathcal{B}\left(\mathbb{R}^{+}\right)
\end{aligned}
$$

Observação 5. A demonstração da Proposição 7 é análoga. Basta definir a sequência $\left(\xi_{-j}^{\prime}\right)_{j \geq 1}$ por:

$$
\xi_{-j}^{\prime} \doteq R_{\dagger}\left(Y_{-j}\right)=-\log \overline{F_{\dagger}}\left(Y_{-j}\right) \quad \text { para cada } \quad j \geq 1
$$

e notar que os recordes da sequência $\left(\xi_{-j}^{\prime}\right)_{j \geq 1}$ ocorrem nos mesmos índices da sequência $\left(Y_{-j}\right)_{j \geq 1}$, já que a função $R_{\dagger}$ é crescente. Assim, a sequência $\left(\xi_{J_{k}(0)}^{\prime}\right)_{k \geq 1}$ é a sequência de recordes de $\left(\xi_{-j}^{\prime}\right)_{j \geq 1}$ e o resultado segue dos mesmos argumentos da demonstração acima.

\section{A.2 Mais alguns resultados utilizados}

Um processo pontual $\Pi$ em $\mathbb{X}$ pode ser interpretado como um elemento aleatório no sistema de subconjuntos enumeráveis de $\mathbb{X}$. Mas existe uma definição alternativa, como medida de contagem aleatória definida por:

$$
\gamma(\omega, B) \doteq \#(\Pi(\omega) \cap B) \quad \text { para cada } \quad B \in \mathcal{X}
$$

onde $\mathcal{X}$ é uma sigma-álgebra de $\mathbb{X}$. Também podemos nos referir a $\gamma$ como processo pontual.

Assim, a intensidade $\mu$ do processo pontual $\gamma$ é dada por:

$$
\mu(B)=\mathbb{E}[\#(\Pi \cap B)]=\mathbb{E}[\gamma(B)]
$$


Fórmula de Campbell. Seja $\gamma$ um processo pontual em $\mathbb{X}$ com intensidade $\mu$. Então $\int h(x) \gamma(d x)$ é uma variável aleatória tal que:

$$
\mathbb{E}\left[\int h(x) \gamma(d x)\right]=\int h(x) \mu(d x)
$$

para cada função $h: \mathbb{X} \rightarrow \overline{\mathbb{R}}$ tal que $h \geq 0$ ou $\int|h(x)| \mu(d x)<+\infty$

A demonstração pode ser encontrada em Last e Penrose (2017), Proposição 2.7, página 13.

O funcional de Laplace $L_{\gamma}$ de um processo pontual $\gamma$ em $\mathbb{X}$ é definido por:

$$
L_{\gamma}(h) \doteq \mathbb{E}\left[e^{-\int h(x) \gamma(d x)}\right]
$$

para cada função $h: \mathbb{X} \rightarrow \mathbb{R}^{+}$.

O teorema a seguir é uma caracterização de processos de Poisson por funcional de Laplace.

Funcional de Laplace para processos de Poisson. Seja $\mu$ uma medida s-finita em $\mathbb{X}$ e $\gamma$ um processo pontual em $\mathbb{X}$. Então $\gamma$ é um processo de Poisson com intensidade $\mu$ se e somente se:

$$
L_{\gamma}(h)=\exp \left[-\int\left(1-e^{-h(x)}\right) \mu(d x)\right]
$$

A demonstração pode ser encontrada em Last e Penrose (2017), Teorema 3.9, página 23.

Dizemos que um processo pontual $\gamma$ é próprio se existem uma sequência de elementos aleatórios $\left(X_{k}\right)_{k \geq 1}$ em $\mathbb{X}$ e uma variável aleatória estendida $\kappa$ em $\mathbb{N} \cup\{0,+\infty\}$ tal que $\gamma$ pode ser escrito na forma:

$$
\gamma=\sum_{k=1}^{\kappa} \delta_{X_{k}}
$$

Neste caso, temos:

$$
\int h(x) \gamma(d x)=\sum_{k=1}^{\kappa} h\left(X_{k}\right)
$$

para cada função $h: \mathbb{X} \rightarrow \mathbb{R}^{+}$.

Daí seguem:

$$
\begin{aligned}
\mathbb{E}\left[\sum_{k=1}^{\kappa} h\left(X_{k}\right)\right] & =\int h(x) \mu(d x) \\
\mathbb{E}\left[e^{-\sum_{k=1}^{\kappa} h\left(X_{k}\right)}\right] & =L_{\gamma}(h)
\end{aligned}
$$

Se $\gamma=\sum_{k=1}^{\kappa} \delta_{X_{k}}$ é um processo de Poisson em $\mathbb{X}$, temos:

$$
\mathbb{E}\left[e^{-\sum_{k=1}^{\kappa} h\left(X_{k}\right)}\right]=\exp \left[-\int\left(1-e^{-h(x)}\right) \mu(d x)\right]
$$

para cada função $h: \mathbb{X} \rightarrow \mathbb{R}^{+}$.

Dizemos que $K$ é um kernel de probabilidades de $\mathbb{X}$ em $\mathbb{Y}$, se a transformação $K: \mathbb{X} \times \mathcal{Y} \rightarrow[0,1]$ é tal que $K(x, \cdot)$ é uma medida de probabillidades para cada $x \in \mathbb{X}$ e $K(\cdot, C)$ é uma função $\mathcal{X}$-mensurável para cada $C \in \mathcal{Y}$.

Sejam $\gamma=\sum_{k=1}^{\kappa} \delta_{X_{k}}$ um processo pontual próprio em $\mathbb{X}, K$ um kernel de probabilidades de $\mathbb{X}$ em $\mathbb{Y}$ e $\left(Y_{k}\right)_{k \geq 1}$ uma sequência de elementos aleatórios em $\mathbb{Y}$ tais que a distribuição condicional de $\left(Y_{k}\right)_{1 \leq k \leq n}$ dados $\kappa=n \in \overline{\mathbb{N}}$ e $\left(X_{k}\right)_{1 \leq k \leq n}$ é de elementos aleatórios independentes com distribuição $K\left(X_{k}, \cdot\right)$ para $1 \leq k \leq n$. Dizemos que o processo pontual:

$$
\hat{\gamma} \doteq \sum_{k=1}^{\kappa} \delta_{\left(X_{k}, Y_{k}\right)}
$$


é uma K-marcação de $\gamma$. Se existe uma medida de probabilidades $\mathbb{Q}$ em $\mathbb{Y}$ tal que $K(x, \cdot)=\mathbb{Q}$ para cada $x \in \mathbb{X}$, então dizemos que $\hat{\gamma}$ é uma $\mathbb{Q}$-marcação independente de $\gamma$.

Denotamos por $\mathcal{X} \otimes \mathcal{Y}=\sigma(\mathcal{X} \times \mathcal{Y})$ a sigma-álgebra produto e por $\mu \otimes K$ a seguinte medida:

$$
(\mu \otimes K)(C) \doteq \iint \mathbf{1}_{C}(x, y) K(x, d y) \mu(d x) \quad \text { para cada } \quad C \in \mathcal{X} \otimes \mathcal{Y}
$$

Teorema das Marcas. Seja $\hat{\gamma}$ uma K-marcação de um processo de Poisson próprio $\gamma$ com intensidade $\mu$ s-finita. Então $\hat{\gamma}$ é um processo de Poisson com intensidade $\mu \otimes K$.

A demonstração pode ser encontrada em Last e Penrose (2017), Teorema 5.6, página 42. 
APÊNDICE A 


\section{Referências Bibliográficas}

Formentin e Swart(2016) Marco Formentin e Jan M. Swart. The limiting shape of a full mailbox. $A L E A, 13: 1151-1164$. Citado na pág.

Guiol et al.(2013) Hervé Guiol, Fábio P. Machado e Rinaldo Schinazi. On a link between a species survival time in an evolution model and the bessel distributions. Brazilian Journal of Probability and Statistics, 27(2):201-209. Citado na pág.

Last e Penrose(2017) Günter Last e Mathew Penrose. Lectures on the Poisson Process. Cambridge University Press. Citado na pág.

Pinheiro(2015) Maicon A. Pinheiro. Processos pontuais no modelo de Guiol-Machado-Schinazi de sobrevivência de espécies. Dissertação de Mestrado, Instituto de Matemática e Estatística. Citado na pág.

Resnick(1973) Sidney I. Resnick. Limit laws for record values. Stochastic Processes and Their Applications, 1(1):67-82. Citado na pág. 